
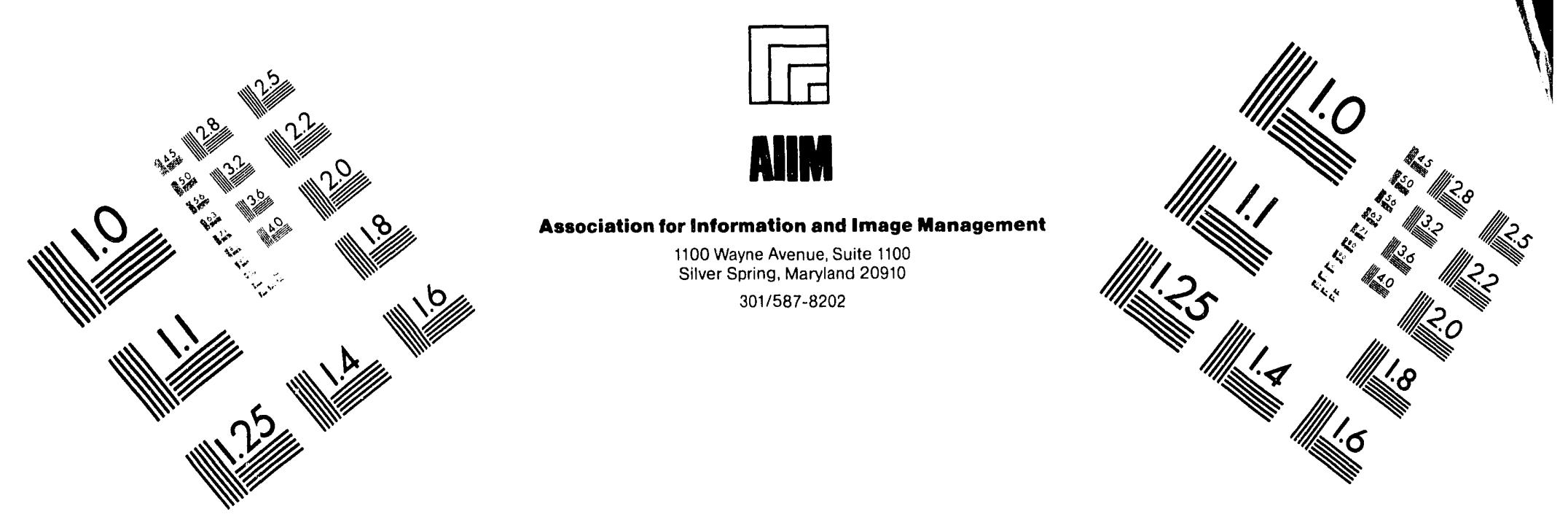

\title{
Centimeter
}

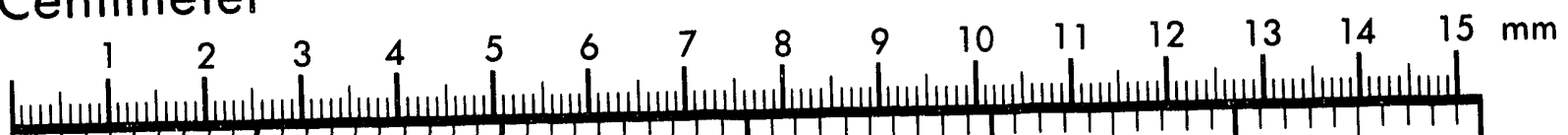

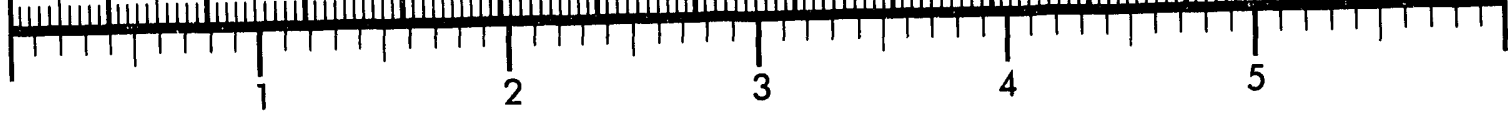
Inches
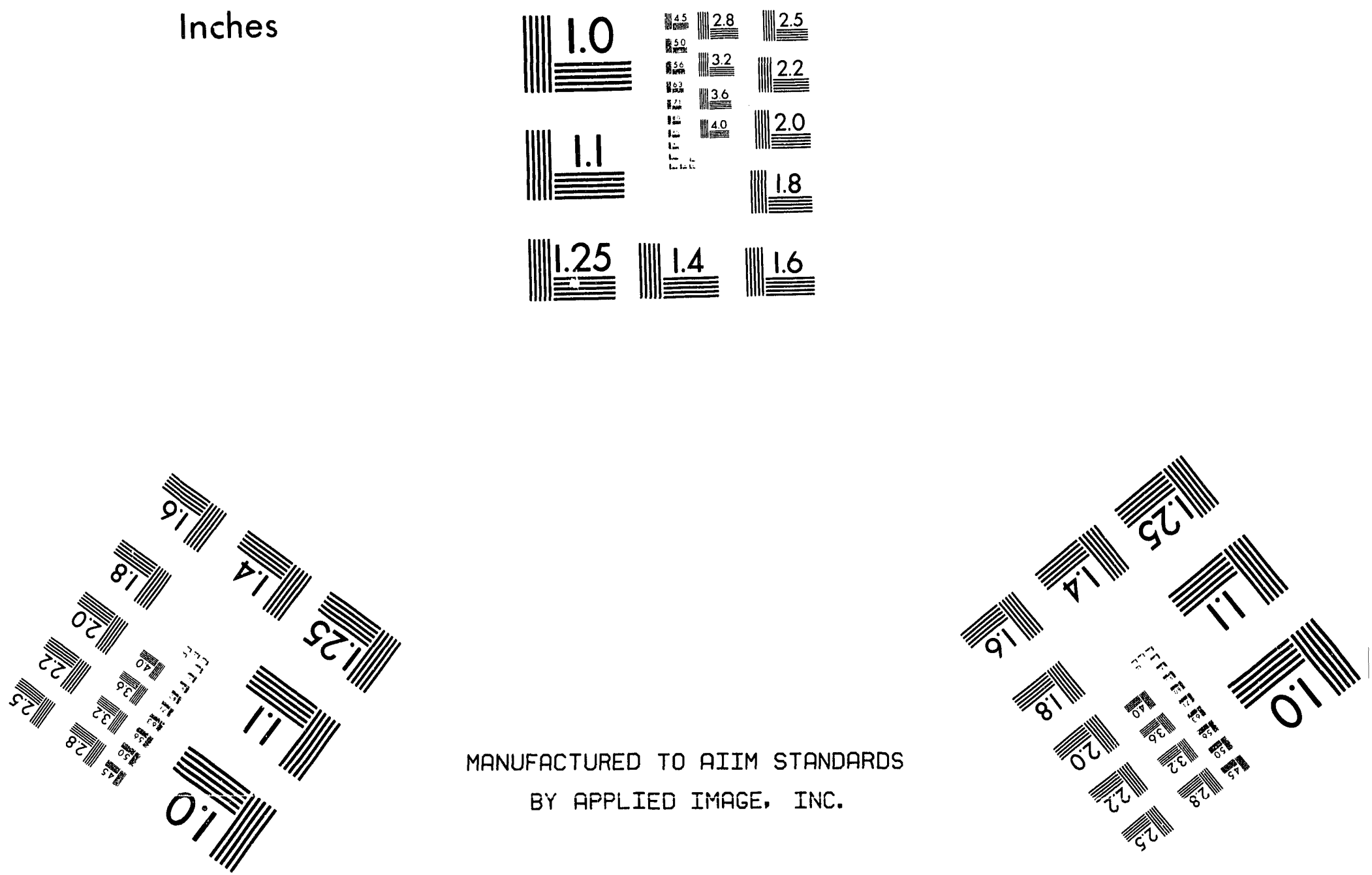

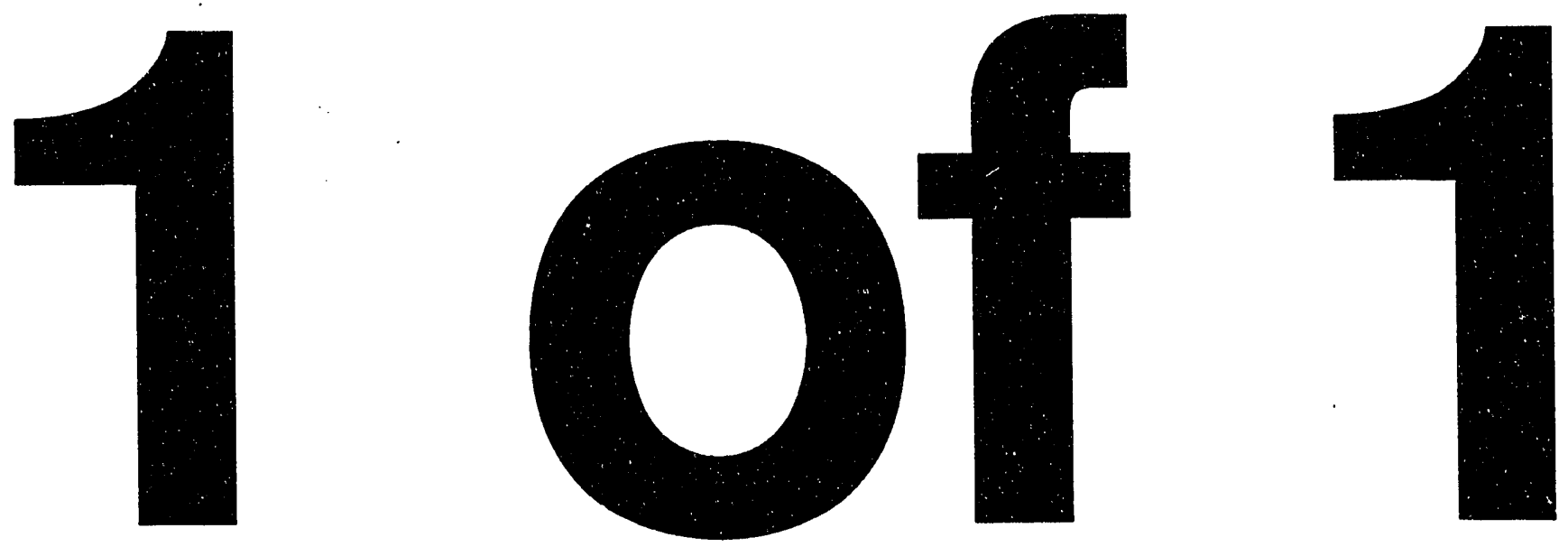


\title{
Information Technology Resources Assessment*
}

\author{
Stewart C. Loken, Editor \\ Information and Computing Sciences Division \\ Lawrence Berkeley Laboratory \\ University of California \\ Berkeley, California 94720
}

January 1993

Prepared for the

Department of Energy Information Resources Management

Long-Range Plan: FY 1994 - FY 1998

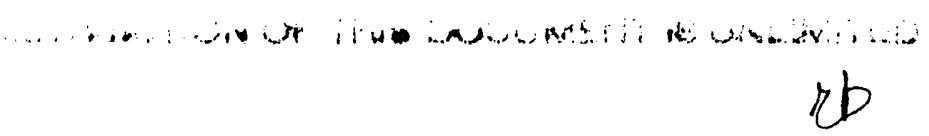

*This work was supported by the U.S. Department of Energy under Contract No. DE-AC03-76SF00098. 


\section{Information Technology Resources Assessment}

\section{Introduction}

Every IT resource is a peripheral on a network.

The products of our information workforce will determine our ability to compete.
Today, the single most important influence on the development of information technology resources is their connection to a network; everything is a peripheral on a network. Personal beepers, personal portable phones, and hand-held computers connect the worker to the network-any time and anywhere-to receive, process, and send information to other peripherals, whether they are workstations, storage devices or supercomputers.

The growing capabilities of networks are attended by increased interdependency among IT resources, as technical advances in one area, such as computational power, create demands in others, such as network speed and storage capacity. This same interdependency ensures the propagation of the newest information technology on a global scale. With the emergence of global information networks, mastery of these tools of the information age becomes an economic necessity. The products of our information workforce and technologists - enabling technologies such as scientific and technological research, workforce education, more sophisticated software, better management, advanced communications, and electronic finance-will determine our ability to compete.

DOE is a leader in the development and application of information technology. Today, the most important prototype of the future global network is the national network that links information systems throughout the DOE complex. Network-based distributed computing and other types of high-performance computing are used within DOE to support its defense programs and energy research missions. DOE has, for instance, developed the ability to carry out computer simulations of complex physical phenomena that are either too dangerous or environmentally destructive for real-world experimentation.

Where these phenomena are too complex for the computer capabilities available today, DOE continues to advance information technologies to meet its mission requirements. Recently, DOE assumed an expanded role in advancing and deploying technologies 
Distributed computing...

Portable and pocket computers and communications systems... when it became a major participant in the President's High Performance Computing and Communications (HPCC) Initiative.

Information technology is also important to DOE as a service organization, facilitating our contact with the public and enhancing our ability to provide information regarding energy strategy, production, and use.

DOE's continuing research and development efforts in information technology will lead to the establishment of needed information resource standards, improve our responsiveness to the information needs of people and organizations, increase our productivity, change our personal and business interactions, and improve our ability to manage information.

This report is divided into a number of sections. Each covers a technology that is significant for DOE programs, describing the technology, its applications, developing trends, timeframes for acquisition and implementation, and significant technical and management issues surrounding its integration into DOE programs. We have attempted to present the material in a cumulative fashion, and so that interdependencies between technological areas are apparent.

We begin with distributed computing (Section 2) because it represents the movement of information technology resources toward networked-based "location-transparent" integration. The power of distributed computing resides in its adaptive and synergistic use of diverse, networked IT resources to solve specific problems. Many programs throughout the DOE have turned to distributed computing to address problems that are too large or too complex for single computers.

It is useful to consider the other technology areas covered in this forecast as elements of a distributed computing environment.

Today, development of all kinds of hardware systems is guided by the need to exchange information with other systems. Rapid developments in portable and pocket computers (Section 3) and communications systems (Section 4) are resulting not only in increased portability, but also in integration of portable computing and communication. For both administrative and scientific computing this means that access to network-based information will 
Workstations and administrative systems...

Hardware for high performance computing...

Data storage systems...

Data communications networks...

Data acquisition and control... be possible wherever people need to work, whether in a research center, a hotel room, or in transit.

Workstations and administrative systems (Section 5) are a fundamental part of the IT environment, having themselves redefined this environment in less than 10 years. Development of these systems will be in the direction of increased interoperability and versatility, as improvements in networks and infrastructure technology lead to heterogeneous distributed computing, video conferencing, multimedia, and other network-based applications.

The traditional vector supercomputer is being overtaken by parallel computing systems (Section 6). Already, massively parallel systems are having an impact on computational science. As parallel systems come into increased use they will be integrated into the emerging distributed computing environment at DOE, particularly in connection with the computationally intensive Grand Challenges (Section 17).

As computational power has increased, so have data storage requirements. Data storage systems (Section 7) are now a critical resource for projects that accumulate large amounts of experimental data, such as experiments in high energy and nuclear physics, and for projects that create extensive models, such as the Global Climate Modeling (GCM) project.

The network (Section 8) is the "glue" that binds everything together. Modern telecommunications systems such as the Integrated Services Digital Network (ISDN) (Section 9), high speed networks for data communication in distributed computing applications, and radio systems for portable computing and communications have spawned a host of new approaches to information exchange and collaborative work. With multimedia and video conferencing (Section 10), the desktop computer is emerging as a desktop communications station as well (Section 11).

Data acquisition and control (Section 12) falls increasingly within the bounds of the distributed computing environment. To provide global data sharing and remote diagnosis of experimental equipment, experiments need to integrate their apparatus into the international network. This way of working is already the norm for high energy and nuclear physics programs. It will also be a necessity at new facilities such as light sources, because it will be impossible to locate 
Software development... Software development (Section 13) is possibly the greatest challenge facing the DOE programs. The growing complexity of scientific applications requires new approaches, such as rapid prototyping, which allow for relatively painless adjustments to changes in the development model.

Scientific databases... New methodologies are required for creating and managing everlarger scientific databases (Section 14). For example, object-oriented database management systems (OODBMS) can greatly simplify systems analysis for complex scientific applications. OODBMS also allow improved interactive data exploration by mapping data structures to the real world.

Scientific visualization...

Virtual reality...

Hypermedia...

Scientific visualization software (Section 15) resolves complex data resulting from large-scale scientific computations into graphic form. It is inherently based in a distributed, heterogeneous hardware environment, typically including supercomputers, mass storage devices and graphics workstations.

Virtual reality (Section 15) optimizes location transparency and the user's interactive control, allowing unmediated interaction between data and human senses.

Hypermedia (Section 16) extends the concept of virtual reality to include movement through information space. Control over data exploration is localized, and the user is empowered to change the structure of the hyperdocument as needed. Management of multimedia and hypermedia documents will become necessary for both education and technology transfer.

HPCC Initiative... The DOE is one of four major Federal agencies involved in the High Performance Computing and Communications (HPCC) Initiative (Section 17). The goals of the Initiative are to extend the country's technological leadership in high performance computing and communications, to hasten innovation in the information technologies in the service of national education and security needs, and to enhance the nation's industrial competitiveness.

Agile manufacturing... We conclude this forecast with a case study in distributed computing. Agile manufacturing (Section 18) is revolutionizing the relationships between engineering design and manufacturing processes, electronically integrating these by means of a distributed computing 
The foundation of distributed computing is communication. system. Agile manufacturing can greatly reduce manufacturing costs and flow-time. We include an example of a DOE project which has extended this technology in a new direction, showing the potential for adaptive reuse which is an essential part of today's IT environment.

\section{Distributed Computing}

This technology assessment reviews advances in many specific areas, ranging from palmtop computers to supercomputers. Just as important as the progression of these individual technologies, however, is the shift that is occurring in the paradigm of how these technologies are used. This emerging paradigm has many names: metacomputing, full-spectrum computing, information systems, highperformance computing, or, as we will call it, distributed computing. Distributed computing involves the logical assembly of networkbased components-including independent computing, storage, and input/output (I/O) elements-to form a "virtual system," a single computational resource optimally configured to solve a specific problem.

The benchmark that distinguishes distributed computing from conventional networked computing is location transparency. All the resources in a distributed environment are part of a single cooperative system. Regardless of the user's location or the location of the resources being used, the distributed system presents itself to him or her simply as "the computer" on which the user is doing work.

The ability to marshal distributed resources-workstations, vector and parallel supercomputers, mass storage systems, special purpose computers such as visualization servers, and specialized softwarewill produce a range of benefits, including new capabilities available through sperialized machines and software, expanded capacity gained through the opportunistic use of spare compute cycles, and enhanced convenience and productivity resulting from improved software.

Distributed computing applications may be structured in several ways. Data can be distributed across several computers, with similar or identical functions being performed by each computer on its share of the data, for example, to remove background "noise" from a data signal. 
DOE facilities are using clusters of workstations for distributed computing.
Alternatively, different functions can be distributed across several computers, all of which operate on the same data, for example, to test different algorithms.

Finally, a pool of users can run multiple applications on a distributed system comprising multiple computers that manages the concurrent jobs in a serial or parallel fashion, much as a single mainframe would.

\subsection{Applicability}

Distributed computing has already gained a foothold in some operational research computing environments, such as the National Energy Research Supercomputing Center (NERSC) and the High Performance Computing Research Centers (HPCRCs). Several DOE facilities are using homogeneous clusters of workstations, that is, workstations based on the same operating systems and communications protocols, for concurrent distributed computing. For some applications, such homogeneous clusters are viewed as a viable replacement for the current generation of vector supercomputers. The power, economy, and scalability of these systems are particularly attractive to industrial users who have computationally intensive applications but cannot afford vector or parallel supercomputers. Also, small groups of users can start with a few workstations and then increase capacity, resources, and capability by adding to the cluster.

Recently, several high-performance heterogeneous computing applications have been developed that run on multiple vector and parallel supercomputers connected by high performance networks. Because heterogeneous supercomputing can provide several resources with differing computational characteristics, it is well-suited to solving large, coupled problems such as ocean-atmosphere models, chemically reacting flow simulations, and thermomechanical engineering calculations.

In the emerging technologies of collaborative engineering and agile manufacturing (see Section 18), distributed computing can integrate and streamline the design, analysis, and manufacturing cycles, thereby coupling simulation tools, engineering databases, CAD facilities, production facilities, and special purpose computers for process control and monitoring. Such applications are now being developed within the DOE complex. 
In a few years we will see low-latency networks for process-toprocess communication.
Efficient maintenance and administration tools are needed to manage distributed resources.

\subsection{Trends and Timeframes}

Current investigations center on heterogeneous distributed computing systems. This approach must accommodate the many different available processors connected to the network. These systems may link massively parallel supercomputers, multiple-CPU supercomputers, or heterogeneous networked workstations. As part of the network communication process, heterogeneous distributed systems must translate data into platform-independent representations. Platform-independence permits development of applications that exploit the full spectrum of computational resources.

The capabilities of a distributed computing environment are inseparable from those of the network that connects the individual components. The next few years will witness development of "lowlatency" networks, such as the HIPPI, ATM, and proposed scalable coherent interface (SCI) standards, that are capable of the fast roundtrip times needed for process-to-process communication. Higherlatency general purpose networks will handle operating and file system communication.

\subsection{Technical and Management Issues}

Advances in network technology, particularly in high-bandwidth, low-latency systems, will be necessary to make heterogeneous distributed computing a practical reality. A reliable production environment will require task assignment methods that account for the character of the computational tasks and the capabilities of available processors. These methods include dynamic scheduling, process migration, and fault tolerance. Dynamic assignment of tasks also raises the problem of ensuring the reproducibility of results when different platforms, using potentially different numerical representations, may be involved each time an application is run. A fully functional distributed environment will also require further development of profiling and debugging tools that include heterogeneous capabilities.

Efficient system maintenance and administration tools will become essential for maintaining an operational system as centralized servers are replaced by distributed resources. For example, cost recovery and security within the DOE complex will become significantly more complex when multiple organizations or laboratories are responsible for the physical resources that make up the distributed computing environment. 


\section{Portable Computing and Communications}

The downsized equivalent of a typical office computing infrastructure costs less than $\$ 6,000$.

Ultraportables will provide access to remote computing and database resources.
Existing PC user interfaces, including DOS, Windows, and Macintosh, are now available on portable computers that, at less than 3 pounds, are smaller and more portable than conventionas laptop and notebook computers. These "ultraportables" can run for 10 to 100 hours on battery power. The addition of other portables, such as phone, fax, PC, modem, 300 DPI printer, and scanner gives one the downsized equivalent of a typical office computing infrastructure for less than $\$ 6,000$.

Recent arrivals in the ultraportable category include palmtop computers like the Apple Newton, Sony Dataman, Poqet PC, and HP95; pen-based systems like the IBM ThinkPad, the GridPad, and the NCR pentop; small, portable cellular phones like the Motorola TAC (flip phone); and portable cellular modems and packet radio interfaces.

\subsection{Applicability}

For sophisticated computer users, ultraportable computing and communications will provide access to remote computing and database resources. The less sophisticated user will gain easier access to communications and e-mail networks. Eliminating typing, cables, and the need to plug into a modem will increase the number of potential users, as will the prospect of transforming rides to the airport, commutes on a train, and waits for appointments into productive work time. In addition to freeing users of spatial constraints, ultraportable technology will allow essentially 24-hour contact with key projects and personnel.

For management and administration, the potential of pocket and portable computing technology is the same for DOE programs as for the business community. We can expect it to be adopted as naturally in the DOE management community as have fax machines and laptops.

For DOE's scientific programs, the combination of ultraportable computing and communications will make it possible for a scientist attending a meeting to retrieve an appropriate journal article or technical image from large remote databases. Wirelessly networked portable devices will commonly be used to control scientific and engineering computations, and users of pen-based systems will input 
equations and technical data over the network. Compact, portable devices will free the scientist from the workstation and the keyboard.

Up to now, development of computer-based scientific journals and computerized library holdings has been thwarted because users have had either to come to the library or sit in front of a workstation or terminal to access the information. Inexpensive handheld, wirelessly networked computers with high resolution displays will give almost anyone access to computer-stored information. Beyond the scientific community, this ease of access will open up a commercial market for information services on a scale vastly greater than at present.

\subsection{Trends and Timeframes}

Laptop and palmtop computers are available now. Fax cards and cellular modem cards are available, but they are expensive and shorten battery life. Laptop-sized pen-based systems have been available for about 12 months, and a new generation of products was released in the fall of 1992. Handheld CD-ROM readers that use special ( 3.5 inch) CDs have also been available for about twelve months. First deliveries of handheld pen-based computers are expected in 1993. The first handheld CD-ROM readers that use standard CDs will be announced in 1993.

During the next 3 to 5 years, laptops will continue to decrease in size.
During the next 3 to 5 years, laptops will continue to decrease in size, limited only by the keyboard and screen size, while their computing power will remain the same or increase. Battery life will improve as a result of better battery technology and new, low-power very-largescale integration (VLSI) designs. As power requirements continue to decrease, we may even see solar-powered notebook computers. Software for ultraportables will continue to come from the mainstream PC marketplace for at least the next five years, or until the ultra-portable market is large enough to support portable-only products. Today, we are just starting to see the emergence of operating system utilities aimed at the portable computing marketplace.

In the same timeframe-the next 3 to 5 years-a separate evolutionary course will unfold, as separate portable technologies are merged into integrated products. For example, the PC, phone, and CD-ROM will be combined into a single small package with a single source of battery power. These designs will also have shared hardware resources and more convenient, compact designs. 
Pen-based portable computers will probably be the next personal computer to have an impact.

The cost-effective application of ultraportable technology is the most significant management issue.
Because of their appeal to a new market, namely, people who do not use keyboards in their work, pen-based portable computers will probably be the next personal computer technology to have a major impact. The widespread adoption of pen-based computers (large or small) will be paced by improvements in the quality of handwriting recognition software. While major improvements can be expected in the next 3 to 5 years, it is not certain that recognition systems capable of performing in a variety of applications will be good enough for consumer use in this timeframe. However, the non-consumer business market for handheld, application-specific pen-based systems will be very large.

Good pen interfaces on systems that support remote wireless data communications will lead to a new generation of applications. Users will be able to exchange handwritten (or computer-transcribed) messages with anyone who has a similar device anywhere on (or near) the planet-in close to real time.

\subsection{Technical and Management Issues}

The cost-effective application of ultraportable technology is the most significant management issue. Not every member of an organization needs a $\$ 5,000$ or $\$ 10,000$ portable computing/communication environment. However, the increased productivity and availability gained by professionals who travel extensively or work at multiple locations can easily justify the cost of laptops, cellular phones, and even portable fax machines.

Apart from adopting portable computing for internal use, many businesses and institutions that serve the public will need to accommodate the new world of portable computing and communications. For example, airlines might provide modem service in flight and allow use of cellular communication on the ground.

The new technology will require that government agencies adjust their security arrangements to allow a person onsite with $\$ 10,000$ of : computer and communications gear in a shoulderbag. Similarly, organizations will need to reassess their computer security arrangements in light of portable radio terminals. In most cases the new technology does not threaten existing security systems; it just removes the issue of physical access control.

Several technical problems stand in the way of producing mature portable systems. New, low-power VLSI technology and improved 
batteries are on the horizon, but systems that can run on batteries for months or years are a long way off, as are flat-panel displays that have both CRT-quality and low power consumption. Areas in which current work shows promise include better character recognition software, more powerful processors, and new algorithms. Finally, as more portable systems come into use with cellular, pocket radio, or PCN communication systems (which are discussed in the next section, Radio Network Technologies), the allocation of EM (electromagnetic) spectrum for portable data communications may have to be increased.

\section{Radio Network Technologies}

Wireless radio networking is finding increasing use for computer-tocomputer, computer-to-person and persun-to-person communication. Many such wireless systems have been developed for military or experimental use. We will discuss only the ones that are now or may soon be in commercial use, namely cellular, PCN, and packet radio systems.

The most widely used radio networking technology is the cellular phone network.

PCN phones are smaller, use less power, and cost less than cellular phones.
The most widely used radio networking technology is the cellular phone network. Cellular phone service is available in most major cities around the world. A cellular phone network consists of a number of radio "cells," each covering a limited geographical region of from 1 to 20 square kilometers. The cells connect portable telephones to each other and to the regular phone system. Limiting the geographical area handled by each cell (that is, increasing the total number of cells system-wide) increases the number of active phone calls a given set of bands can service system-wide. As a user migrates from one cell to another the portable phone and the cellular network "hand off" the call from one cell to the next, maintaining a path, or circuit, between the cellular phone and the destination. For this reason, cellular networks are called "circuit-switched" networks.

Cellular phones work like normal phones in the way calls are placed and received, and can be attached to modems. A portable computer equipped with a modem and cellular phone can be connected to other computers just as if it were a wire-based phone. The bandwidths and modem speeds are also comparable to those available on wire-based phones.

Handheld cellular transmitters and their batteries must be relatively powerful, which adds to their bulk and cost. This limitation is compounded by the fact that cells smaller than about a square kilometer are impractical. A system now under development, PCN 
Iridium would allow most places on the earth to communicate with at least one satellite. (personal communication network), overcomes most of these limitations. In the PCN system the cell size is much smaller, often as small as a city block. This allows the phones to be smalier, use less power, and cost less than cellular phones.

PCN and cellular systems are not compatible and, to be used in both systems, any single phone would need redundant electronics and logic. However, PCN and cellular networks handle digital computer communications in similar ways.

Cellular phone systems have a maximum as well as a minimum cell size and, though most major metropolitan areas have cellular phone service, most of the nation and world do not have the user density to justify the investment in cellular transmitters. Motorola is developing a satellite-based system that would extend cellular phone and computer communications to nearly the entire surface of the earth. This system, called Iridium after the element having 77 electrons, would have 77 small communications satellites, orbiting the Earth in polar orbit so that most places on the surface can communicate with at least one satellite. Iridium works like the cellular phone system except that both the user and the transponder can move, with the satellites handing off calls as one moves out of range and another moves in.

Cellular, PCN and Iridium are circuit-switched networks. This means that $100 \%$ of the available bandwidth is used for the duration of each call. However, the normal phone system uses bandpass filters to limit the available bandwidth to about $5 \mathrm{Khz}$ per channel.

The other major type of radio network is the packet-switched network. Packet-switched networks break up the data stream into units of data called packets. These packets are interleaved with other traffic in the network and sorted out at the destination. So, rather than switching the circuit, the network routes the data by labeling it with its destination and switching it on the fly. This is how most digital computer networks work. Packet radio works by using the same frequency (or radio channel) for a number of stations (computers or communications devices). These stations communicate by breaking up their data into small units and transmitting these units only when no one else is transmitting. They can use this mechanism to broadcast the same data to a number of selected stations. 
Packet-switched

networks support larger

bandwidths than

cellular networks and

are less limited in

range.

Handheld or pocket computers will use wireless networks to access databases.

In major U.S. cities, $P C N$ service should start in the next few years.
Packet-switched radio networks can support larger bandwidths than cellular networks and are less limited in range. They can be large, servicing up to 2500 square kilometers, or small, working only within the office environment as a replacement for conventional copper-wire networks. In general, packet radio systems are dedicated to special uses such as police information systems and private networks.

\subsection{Applicability}

For a user on travel or stationed at a remote site, cellular modems greatly improve access to e-mail and files, and allow integration of home systems with office and laboratory environments. Wireless LANs, a technology related to the packet radio, allow computers to be moved easily while maintaining full connectivity, bringing new flexibility to project and field site environments.

The limited EM spectrum allocated to packet radio networks restricts them to a complementary role, significantly extending the possibilities of remote and distributed computing, but not replacing the National Research and Educational Network (NREN) or other high-speed networks.

In the foreseeable future, handheld or pocket computers will use wireless networks to access databases, particularly digital libraries and bibliographic resources, from virtually anywhere. Another common use may be the cooperative production of documents.

\subsection{Trends and Timeframes}

Cellular networks and phones are widely available today. The phones cost from $\$ 100$ to $\$ 1000$ and air time costs from $\$ .05$ to $\$ 1.00$ per minute. To be used on a cellular network, a computer generally requires a modem interface that generates the "dialtone" and other signals that most modems expect from the phone system. These interfaces cost about $\$ 300$ and can be used with any modem that has a RJ11 plug cable.

PCN systems are being deployed in many countries, and Motorola has developed a prototype combo phone that can automatically switch between PCN and cellular service transparently to the user. In major U.S. cities, PCN service should start in the next few years, primarily near airports and in office districts, where existing cellular systems are overloaded. PCN-only phones will cost less to purchase than cellular phones, and the cost of air time will be comparable to existing wire-based phone service. 
Wireless LAN

technology permits

communication with

portable terminals over

an area the size of $a$

building or campus.
Security guidelines and encryption schemes will have to be developed and applied.
Iridium could start service as early as 1997 . The main difficulty is launching and maintaining a large fleet of satellites in low earth orbit. Initially, in the 1997 timeframe, handheld phones that can communicate with these satellites will be comparable in price and size to today's smallest cellular phones. Computer use of these satellites will be as easy as voice phone service.

A technology related to the packet radio, the wireless LAN, is currently on the market. It can be more cost-effective than coaxial cable, particularly in environments where dynamic reconfigurability is important. Wireless LAN technology permits communication with portable terminals over an area the size of a building or campus. A wireless LAN allows a workstation or fileserver to be moved without having to be recabled. In addition to distance restrictions, wireless LANs have a limited bandwidth, typically less then 1 megabit per second.

Packet radio networks and wireless LANs are in operation today, using hardware available from a number of vendors. Within the next few years packet radio service will have been established in most commercial cellular phone regions. A typical packet radio system provides 56 kilobit-per-second service within 60 kilometers of the central transponder, sufficient for $\mathrm{TCP} / \mathrm{IP}$ networking to remote locations and homes. Network adapters are available to connect packet radio systems to local area networks. Portable packet radio interfaces will become available in the next one or two years and may become common options on laptop and handheld computers within five years.

\subsection{Technical and Management Issues}

Packet radio and cellular systems are not secure. Anyone with the proper radio receiver can intercept and record network traffic. Cellular modems are particularly vulnerable because common police scanners can be converted to receive cellular traffic. Since audio frequencies are used in the modems, ordinary tape recording equipment can record a user session. To protect confidential data security guidelines and encryption schemes will have to be developed and applied.

Nevertheless, the benefits of radio network technology will almost certainly outweigh the cost of developing security systems. Each organization should determine how it can best use this new technology to improve its competitiveness and use of existing 
information resources, and to promote the development of new resources.

\section{Workstations and Administrative Systems}

Workstations and administrative support computing generally provide tools designed to enhance the performance of individual users or work groups. Intel and Motorola component designs dominate this technology, although other architectural designsgenerally speaking, workstations-are used extensively in engineering and computationally intensive applications. There are various types of workstations, but a "generic" workstation has traditionally been viewed as operating in a UNIX environment. Similarly, the names IBM and Apple are generally taken to delimit the realm of personal computing (although high-end versions of personal computers can be workstations).

Less universally acknowledged is the important role played by the developers of easy-to-use applications software-for instance, Microsoft and Lotus-in establishing the worldwide ascendancy of personal computers. Word processing, spreadsheets, automated printing and publishing, computer aided design and manufacturing (CAD-CAM), computer aided engineering, and mathematical analysis are just some areas for which personal computing now seems indispensable.

\subsection{Applicability}

The next 3 to 5 years should see less new hardware and more complementing technologies.
It is difficult to predict the future of a technology that, in less than 10 years, has grown from nothing into an essential tool for large and small businesses. However, if PCs and workstations follow the pattern of mainframes and minicomputers, the next 3 to 5 years should see less new hardware technology and more emphasis on using complementing technologies. For example, the distinctions between personal computers, workstations, servers and mainframes will diminish as networked client/server computing becomes a standard practice. Although packaging and physical density limitations will restrict the amount of local disk storage feasible on an individual workstation, laser-based storage and other new storage technologies will allow rapid access to a variety of data types, such as image and multimedia data, in addition to character-based information. 


\subsection{Trends and Timeframes}

Aggressive competition may reduce the number of suppliers for Intelbased systems.

Workstations and administrative systems will become scalable.
New operating system and network developments will require newer processors and increased storage capacities.
From 1993 to 1995 will be a period of "shake out" among hardware and software firms. Aggressive price competition is likely to reduce the number of suppliers for Intel-based systems, while Macintosh and UNIX systems will become increasingly important for administrative applications. Toward 1995 Intel, Macintosh, and UNIX systems will become closely integrated as network operating systems come into use, and hardware selection will be largely based on application requirements.

Over the next 5 to 7 years product availability will determine the growth of workstation and distributed administrative applications. In the mid-1990's, workstations and administrative systems will become scalable, so that a business can acquire and add to its system as needed. However, these acquisitions will be contingent on the availability, functionality, and pricing of infrastructure software such as an operating system, network, database, and non-character data manipulation software. Although it is likely that more evolved software engineering practices and object-oriented development languages will result in significant improvements in infrastructure software, development-to-market time intervals will not improve significantly, because software will be exponentially more complex.

\subsection{Technical and Management Issues}

In the 1980's the computer began its move from the central computer facility into the technical and administrative office. This change required large investments in new capital equipment and even greater expenses in personnel training and development. In many cases, productivity improvements were disappointing and expenses for ongoing maintenance and upkeep greatly exceeded original estimates. Although this experience recommends a conservative approach toward replacing and upgrading the installed system base in the 1990 's, new operating system and network developments will require newer processors and increased storage capacities that exceed the capabilities of the installed base. 


\section{Hardware for High-Performance Computing}

A revolution is in progress, based on microprocessor technology, that will determine supercomputer architecture for the next decade.
For the past decade, high-performance computing, the highestcapability scientific computing, has relied on vector supercomputers. Vector supercomputers have a small number of central processing units-16 or fewer-and provide megaflops (millions of floatingpoint operations per second), gigabytes (billions of bytes) of dynamic storage, and megabits (millions of bits) per second in data communications. Today, a revolution is in progress, based on microprocessor technology, that will determine supercomputer architecture for the next decade. Massively parallel computers that have hundreds or thousands of central processors with teraflop, terabyte, and gigabit capabilities are a reality.

It was the microprocessor that made possible not only the personal computer revolution, but also the computerization of consumer products as diverse as programmable video cassette recorders and automobile fuel systems. Because of this commercial success, the economic incentive has been tremendous to improve microprocessor design and also advance the underlying fabrication technology, so that more and more transistor circuits can be placed on a single chip. Today, the size of individual features on commercially produced chips is on the order of 0.8 microns, and an entire central processor with a peak speed of between 120 and 150 megaflops fits on a single chip. This speed is a little more than one-half that of the central processors available in conventional supercomputers in 1985. Microprocessors can frequently provide performance within a factor of ten of a conventional supercomputer, even though memory access speeds, the other principal determinant of supercomputer performance, are still somewhat slower in microprocessor-based computers.

Today, companies such as Thinking Machines Corporation, Kendal Square, Intel Supercomputer Division, Cray Research, NCube, and others are producing massively parallel computers with arrays of hundreds or thousands of microprocessors, or computing "nodes," each of which has its own memory. The main difference between the architectures used by different vendors is in the communication and networking algorithms used between nodes: 
Many applications

should be able to use

massively parallel

computers within two

years.

Memory for

microprocessor-based

devices is becoming less

expensive.
Massively parallel computers are more difficult to program than conventional computers because computational problems must be divided into portions that run on hundreds of nodes simultaneously. Nevertheless, great progress has been made in devising programming strategies and developing the user software environment, and a large fraction if not a majority of applications should be able to use these machines within two years.

An important reason for the emergence of massively parallel computers in scientific computing is the increased speed of the individual nodes. Today, a computer program that temporarily makes use of only one node is still running at a significant fraction of conventional supercomputer speeds. Moreover, the microprocessor revolution has not yet run its course. New fabrication techniques will allow a reduction in the size of a single feature to 0.2 microns or less by the year 2000 . Tenfold or greater increases in performance will accompany these advances.

Peak speeds of massively parallel computers (the aggregate of the speeds of all nodes) are already several times those of conventional supercomputers. As software has improved, increasing numbers of scientific applications have attained larger fractions of the peak speed. Before the year 2000 routine performance will be 1000 times that of current supercomputers, with routine computational speeds of teraflops (10 to the 12th floating point operations per second) for scientific applications.

Meanwhile, memory for microprocessor-based devices is becoming less expensive. While it is current practice to provide from 2 to 32 megabytes of memory on each node (up to 4 million double-precision floating point numbers), these quantities will increase tenfold in three to five years. A computer with 1000 such nodes would have roughly 100 times the memory of the largest supercomputer now available. These increases in speed and memory will fundamentally change the kinds of science and engineering that can be done with supercomputers.

\subsection{Applicability}

Increases in computational power have already charted the future courses of many scientific and engineering disciplines. For example, three-dimensional fluid dynamics modeling will supersede twodimensional modeling in the design of aircraft, submarines, automobile combustion systems, and a variety of other practical 
devices. Accurate electronic structure calculations, currently possible only on relatively small quantities of atoms, will be able to predict the properties of new materials such as exotic metal alloys and plastics. Now in its infancy, molecular modeling of biologically active molecules and of the interaction between drugs and substrate molecules will become commonplace. In molecular biology, molecular dynamics calculations will be possible on systems with 10,000 atoms, such as a small virus.

\subsection{Trends and Timeframes}

In fiscal 1993 massively parallel computers with 100 to 150 gigaflop peak performance will be available from at least three vendors. However, networking performance between the nodes varies between machines, and both operating system software and user-written application software are far from mature. Nevertheless, on the Thinking Machine and Intel offerings, maximum sustained speeds on a few real scientific applications range between 7 and 30 times that of conventional supercomputers.

\subsection{Technical and Management Issues}

A judicious transition to this class of machines is only prudent.
The consensus of the scientific and engineering communities is that massively parallel computing is the only known path to teraflop computing, but how fast should one acquire this technology? While peak performance of these platforms already exceeds that of conventional supercomputers, further improvements will come rapidly. Because the cost of a configured system is in the $\$ 25$ million range, a judicious transition to this class of machines is only prudent. Smaller parallel processing computers having fewer nodes (fewer than 100) cost less. It is essential to distribute these smaller machines so that the maximum impact on scientific programs is realized.

Because of their larger memories and higher speeds, these machines will require greatly expanded mass storage facilities (see Section 7, Data Storage Systems) if they are to be integrated into DOE's emerging distributed computing environment. Even then, if they are used efficiently the volume of data they produce will challenge the capabilities of the existing national network (see Section 8, Data Communications). 
In single calculations, parallel supercomputers can use up the entire storage capability of the largest supercomputer centers.

Storage systems for high performance computing...

The wide acceptance of HIPPI switches and networks has fundamentally changed thinking about storage.

\section{Data Storage Systems}

Over the last five years, the increased capabilities of supercomputers and workstations have caused a dramatic increase in data storage requirements, with respect both to capacity and speed of access. Current-generation vector supercomputers have two to four times the memory of previous generations-about 50 to 150 gigabytes of conventional disk - which it can access at about 3 to 5 megabytes per second. Current parallel supercomputers have eight to sixteen times the memory, with factors of 100 probable in the near future. Their increased memory and speed allow these machines to produce enough data in single calculations to use up the entire storage capability of the largest supercomputer centers, whose long-term "mass" storage-consisting of robotic silos of cartridge tape-provide 2 to 20 terabytes of storage, also accessible at 3 to 5 megabytes per second.

The use of workstations in distributed computing environments has increased workstation memory requirements, and workstation groups too may now have more memory than some current supercomputers.

The most recent development in high-performance storage systems are redundant arrays of inexpensive disks (RAID). Available from several vendors, RAID allows read and write access to eight or more disks in parallel. RAID devices operate at 50 to 60 megabytes per second. Currently, RAID disks are being installed at existing facilities. Within two years almost all disk systems attached to large computers will be of the RAID type.

Low-performance versions of helical scan tape are now available. Helical scan tape systems achieve higher data density by recording the data diagonally across the tape. This is the same concept used in the ordinary home VCR. Packaged in cartridges in conventional tape robots, helical scan tape has a data rate of about 15 megabytes per second and provides access speeds roughly five times faster than current production tape storage.

The wide acceptance of high-performance parallel interface (HIPPI) switches and networks at the high-performance computer centers has brought about a fundamental change in thinking about storage. HIPPI provides transfer rates of 100 megabytes per second, along with the ability to switch data directly between sources and sinks. Current data storage hierarchies have a linear organization, in which 
the collected data passes through a mainframe computer and disk storage before it goes to a tape storage device.

To make network-attached storage system technology a reality, a high-speed network for the direct attachment of client machines is required, along with fast, large-capacity storage devices and proper: software for file management, storage management, device management, and data transfer.

A number of production-quality alternatives will emerge in the next two years. While standards and details remain to be settled, DOE laboratories and vendors are at work on the hardware and software for such systems (see Section 14, Scientific Database Management Systems).

HIPPI switching provides the high data transfer rates needed for direct transfer of data between client machines and storage device:s that are attached to the network. These high transfer rates and accompanying lower hardware costs allow implementation of more powerful data storage systems.

Within the coming year, we will see prototypes or commercial versions of several new storage devices that are suitable for networkattached environments, including

- Solid state disks. These are expensive, but fast. With data transfer rates of 100 megabytes per second, they are limited by the HIPPI network speed.

- RAID disk array. With rates of 50 to 60 megabytes per second, RAID systems are appropriate for supercomputer applications and as network servers.

- Helical scan tape in robotic silos. Vendors predict that sin.gle silos will hold 250 terabytes, accessible at rates in excess of 15 megabytes per second. The robotic silo technology itself will be similar to current devices, but the tape and tape drives will be helical scan devices. Helical scan systems are now available in 4- and 8-mm technologies. A $19 \mathrm{~mm}$ version will soon be available.

- RAID arrays of workstation level disks. This scaled-down version of RAID has a data transfer rate of about 10 megabytes per second and uses SCSI (small computer standard interface) disks, the least expensive disk technology. 
Network-attached

storage system applications include Grand Challenge and national security problems.
Storage systems for distributed computing.
- Optical disk robots (jukeboxes). These provide storage of from 0.5 gigabytes to a few gigabytes at data rates of less than one megabyte per second.

The most immediate applications for network-attached storage system technology are the Grand Challenge and national security problems. Massively parallel, large-memory computers and highspeed cooperative processing networks allow researchers to execute large-scale codes, generating from tens of gigabytes up to several terabytes of data. These quantities are one to two orders of magnitude greater than what the best data storage systems can presently handle. In addition, future data acquisition systems will also create a demand for larger data storage systems.

Looking into the future, vendors describe plans for still greater improvements, based on relatively inexpensive technologies such as

- Optical Tape. Each tape will hold a terabyte and provide data rates of 3 to 4 megabytes per second.

- Striped Tape. Similar to a RAID disk in concept, striped tape will use arrays of tape rather than of disks. Transfer rates are expected to be between 50 and 100 megabytes per second.

- Holographic storage. Still a gleam in the eyes of vendors, this technology will provide transfer rates in excess of 100 megabytes per second and capacities in the multiple terabyte range.

These devices, used in various combinations, will bring the storage system capability of supercomputer systems up to the petabyte range (1000 terabytes) within four or five years. Access speed will be twenty times that of today's conventional (non-RAID) disks.

In addition to their uses for high performance computing, many of these storage systems are being or will be marketed for the workstation environment. These include

- Tape robots for $8 \mathrm{~mm}$ tape, which store 0.5 terabyte or more. These are already available.

- RAID disk arrays based on SCSI disks, which are also currently available. The cost of these systems approaches $\$ 100,000$ but should fall over the next two or three years. 
The distributed file system is a global file system visible to the workstation user.
The transition to RAID disk technology is already in progress.
- Optical disks, which are already becoming a standard for the distribution of software. Their high capacity makes them attractive for this application, despite their relatively slow speed.

The most significant change in the storage environment for distributed computing is the distributed file system, which is a global file system visible to the workstation user. Software such as AFS (Andrew File System) and its competitors allow files, when referenced, to migrate from distant facilities to the computer on which they are needed. Integration of supercomputer and workstation file systems is expected to continue over the next two to three years.

\subsection{Applicability}

Work on many of the Grand Challenge problems, as well as other research areas, is limited equally by shortcomings in computer speed and storage capability. A single climate model calculation, for example, can produce 20 terabytes of data. The ability to access these data at a rate that allows analysis and interpretation is essential.

Large experiments such as the Superconducting Super Collider and the Earth Observing Satellite require terabytes of storage and will soon require petabytes. The required rate of access for these applications varies, but, without exception, current speeds are inadequate and will require both the hardware solutions addressed here and the software solutions addressed in Sections 13 and 14.

\subsection{Trends and Timeframes}

While conventional cartridge silo capacity can be increased by a factor of two by using better data storage methods, the transition to RAID disk technology is already in progress, and the relatively inexpensive $8 \mathrm{~mm}$ tape robot technology is also finding wide acceptance.

Helical scan tape robots are available and new models will soon be in production.

On very high-end workstations, SCSI disks provide storage in the tens of gigabytes. RAID disks based on SCSI technology are beginning to penetrate this market.

HIPPI network switching components are commercially available, and most supercomputers have HIPPI interfaces. Scientific workstations such as the IBM RS/6000 are also beginning to have 
As the global file system emerges, security becomes increasingly important.
HIPPI interfaces. HIPPI-attached RAID-3 and RAID-5 disk arrays are now available.

Hardware systems available in 1993 may be sufficient to implement network-attached storage system technology. However, these systems cannot fulfill the desired performance and reliability criteria.

\subsection{Technical and Management Issues}

The massively parallel supercomputers used for Grand Challenge and national security problems require both network-attached storage and a fast, reliable scientific data management system (see Section 14) that can retrieve specific data from the large quantities of data stored.

Large amounts of data are generated by supercomputers in extensive calculations, but it is ultimately necessary to view and analyze the data on workstations. High-speed networking will provide longdistance access to storage at higher speeds than many workstations can achieve when accessing their local disks. The software for distributed file systems exists today and will continue to improve to the point where a global file system will be technically possible. However, the software for distributed file systems allows data to migrate over the network. This raises security concerns, particularly since this software often provides the easiest path for computer breakins. As the global file system emerges, security becomes increasingly important, especially for the purpose of verifying the identity and rights of a remote workstation or user.

\section{Data Communication Networks}

Data communications involve the transfer of information between components on a network. In recent years this term has come to signify digital networks that connect various types of computers in local, metropolitan, and wide area networks (LANs, MANs, and WANs). Computer networks consist of physical hardware and software protocols. The degree of locality of the service (LAN, MAN, or WAN) affects the latency, which is the round-trip time spent waiting for the response to a communication. In addition to locality, data communications networks are classified by their data transmission capacity, or bandwidth, which is currently in the range of 1 to 100 megabits per second. 
The LAN is the most common type of network.
Workstations using FDDI can achieve throughputs of up to 40 megabits per second.
HIPPI is a good communications medium from supercomputer to supercomputer.

\subsection{Applicability}

The LAN is the most common type of network. It can connect a full range of $\mathrm{IT}$ resources, such as personal computers, workstations, servers, and supercomputers, and can support file transfers, networked file sustems, remote procedure calls, and similar functions.

Ethernet is the most cummon LAN for science and engineering, and is also the most universally accepted data communications standard worldwide. C'onpetition among the many vendors has dramatically reduce. 1 prices for hardware and software, to the extent that Ethernet hardware is now offered for no extra charge in some PCs.

Ethernat has a bandwidth of 10 megabits per second, though some fiber-based implementations operate at higher speeds. For LANs that need to attain higher speeds, the Fiber Distributed Data Interface (FDDI), a fiber-optic data communications ring with a bandwidth of 100 rnegabits pes second, has become the primary choice.

Workstations using FDDI can achieve throughputs of up to 40 megabits per second, making FDDI a good solution for scientific applications that require speeds greater than Ethernet can deliver. FDDI can support medium-speed multimedia applications and is a good candidate for client-server communications for applications invoiving large databases. FDDI is commercially available on twisted-pair copper wiring as well as on fiber optics. This appreciably decreases installation، costs of FDDI, since existing cable plants dn not have to be replaced.

Switched data networks provide full data rates to all connections, because the network traffic for the various connections does not have to share media bandwidth. However, these networks do require high-speed switching elements.

HIPPI, which we have already introduced in relation to storage systems, is an 800 megabit-per-second to 1.6 gigabit-per-second parallel channel. It was designed to supply data communications over a LAN for applications on the scale of Grand Challenge problems and for scientific visualization (see Section 15). When used with copper wiring, HIPPI is limited to a total length of 25 to 50 meters. However, a length of several kilometers is possible with fiber optics extenders. HIPPI is a good medium for communications from supercompuier to supercomputer and from supercomputer to a high- 
ATM is the latest and possibly the greatest standards proposal in the data

communications world. performance storage system within the confines of a computer facility.

Wide-bandwidth communication over greater distance is still several years away, except in testbed applications. Fiber Channel, a followon to HIPPI, will support longer distances, speeds from 100 to 800 megabits per second, and a larger variety of services. Fiber Channel may be used most heavily for communication from mainframes to peripheral local networks.

ATM is the latest and possibly the greatest standards proposal in the data communications world. Touted by the telcos and others as a way of turning data communications into a ubiquitous utility, ATM uses 155-megabit to 622-megabit-per-second switches to route very small data packets efficiently over a variety of media. An ATM switch can be used to route many channels of data, voice, and video simultaneously at speeds ranging from 45 megabits per second to 2.488 gigabits per second on a synchronous optical network (SONET) physical layer.

The telcos are currently planning to offer WAN ATM service in the late 1990s. Most DOE WAN activity during this same period will use ATM at bandwidths from T3 up, since DOE purchases WAN service from the telcos. This service would allow cross-country connectivity between networks at speeds much greater than currently possible.

Other data networks are being developed that are optimized for the high data rates associated with process-to-process communications. These include scalable coherent interface (SCI), distributed shared memory, and reflective memory, which perform the same function for a distributed computing system that an internal communications system, or "backplane," does for a stand-alone computer. These technologies are intended to support data rates ranging from 100 megabits to one gigabit per second.

MAN and WAN data communications will be based primarily on ATM switching fabrics, SONET communication links, and, to a lesser extent, switched multimegabit data service (SMDS). The trend is toward purchasing service from the telecommunications companies, rather than installing privately-owned physical media and equipment. Bandwidth for telecommunications services will range from tens of megabits per second to gigabits per second. In the near term, MAN data communications at bandwidths of less than 100 
The Internet connects universities, government laboratories and agencies, private companies, and other entities.
A major factor in Ethernet is its low installation cost. megabits per second may also be provided by cable television operators by multiplexing available broadcast channels.

Numerous commercial WANs are supported by the public telecommunications companies. The largest primarily noncommercial WAN is the Internet, of which the Energy Sciences Network (ESNET) is a major component. The Internet has evolved into a comprehensive system that connects universities, government laboratories and agencies, private companies, and other entities. The Internet consists mostly of 1.5 megabit-per-second $\mathrm{T} 1$ communication links. Planned upgrades in ESNET will provide T3 service (45 megabits per second) to many DOE sites.

Many DOE laboratories are also involved in high-speed network research through their participation in various national testbeds. The purpose of this research is to demonstrate distributed computational, visualization, storage, and teleseminar applications that are anticipated for the next generation of network technology.

\subsection{Trends and Timeframes}

Data communications technologies available today include some that are mature and several that are evolving rapidly. They span a throsighput range that covers many orders of magnitude. A physical infrastructure designed to support data communications can be obsolete by the time it is installed.

Technologies currently in use at DOE laboratories cover a wide range that includes RS-232, Ethernet, FDDI, HIPPI, and custom equipment. However, the demand for enhanced speed, reliability, and services is strong, and can only be satisfied by new technologies.

However, where cost is a factor and throughput of 10 megabits per second is adequate, Ethernet will continue to be the data communications media of choice. A major factor in Ethernet is its low installation cost, owing to the fact that twisted-pair telephone lines can be adapted to Ethernet. $\because$

Because of Ethernet's popularity, many software packages are now shipped "network ready." This trend is likely to grow more prevalent. and use of Ethernet and other networking technologies will continue for many office and technical environments within the DOE complex for the foreseeable future. 
FDDI will replace

Ethernet in situations where greater speed is required.
ATM networks will

lead to a standard interface between LAN and WAN environments.
As local area data communications progress towards higher speeds through switchable fabrics such as HIPPI, vendors are beginning to develop supporting hardware and software, and some initial offerings are available. However, complete solutions must wait until late 1993 and may be irrelevant if HIPPI and FDDI are eclipsed by the accelerated pace of work on ATM.

FDDI is becoming a mature data communications standard for local area networking and has reasonably good vendor support. Unit: prices are high and reliability low, but prices are expected to fall substantially and reliability to rise as demand grows over the next few years. FDDI will replace Ethernet in situations where greater speed is required and serve as a "backbone" to connect many Ethernet LANs together. Like HIPPI, FDDI could soon become obsolete if ATM lives up to its advanced billing.

The telecommunications companies are taking the lead in adapting ATM for the next generation of high-bandwidth MANs and WANs.

Some ATM equipment is already available at a high cost, and local area network ATM solutions should be available in 1993. Vendors other than the telcos are announcing development of ATM hardware at a dizzying pace, and many believe that this is the wave of the future in data communications.

It is not yet clear whether supercomputer vendors and suppliers of supercomputer connectivity will support ATM, and local area ATM networks for supercomputers may not become available in 1993.

Deployment of small ATM switches should begin by the end of 1993, first by $R \& D$ enterprises that need full-motion video and high-speed data network services.

Ethernet and token ring networks provide data services only. As a need for multimedia (voice, data, image/video) services arises, a migration path will be established towards ISDN and BISDN services (see Section 9).

\subsection{Technical and Management Issues}

As LANs progress toward higher speed and lower latency switching fabrics, and as WANs move toward higher speed switched service, there will be an increasing need to define efficient and standard LAN/WAN interfaces. This necessity may also be felt at individual sites, since the requirement for transferring information between 
ISDN is a global, public network providing voice, data and video services. high-speed, distributed computers will require a standard data communication protocol. Local area ATM networks represent a solution that will lead naturally to a standard interface between LAN and WAN environments.

What procedures should be used to reserve and pay for telecommunications bandwidth is a major management issue. Highpriority or high-bandwidth service may need to be secured with guaranteed minimum pricing, while traffic with a lower priority may be provided on a pay-per-use basis. Another important issue that is becoming increasingly relevant is protection, via encryption or authentication methods, of private data traveling over public networks.

Managing an Ethernet network is itself a management issue. Tools to help the network manager are only now beginning to become sophisticated enough for the large networks that pervade the DOE complex. More complete tools are a year or more away. Security on Ethernets has been for the most part an afterthought. Solutions for Ethernet security, such as labeling, have been proposed but not yet settled on. Current hardware implementations of security are haphazard at best, and good solutions on multiple platforms are several years away.

However, given that procurements for such devices involve long lead times, network management tools and LANs using ATM technology bear consideration in this planning cycle for the more demanding applications.

\section{The Integrated Services Digital Network}

In 1986 the telephone companies (telcos) began field trials of Integrated Services Digital Network (ISDN) technology. Today we are seeing widespread implementation, extending the possibility of digital network service to every desk and every home. ISDN is the only global, digital, public network that can provide voice, data and video services in a single multiplexed connection.

The present implementation of ISDN uses the existing infrastructure of digital switches and the copper twisted-pair distribution system. Digital voice and data service is provided on two circuit-switched " $B$ " channels at 64 kilobits per second each. An additional packetswitched " $D$ " channel provides signaling and other data service at 16 
Frame relay and SMDS

represent a change to switched-bandwidth, on-demand service.
Frame relay networks are used where cost is a factor and low data rates are acceptable.
By the end of 1992 about 1000 telephone company switches had ISDN service. kilobits per second. ISDN is the first step towards implementation of integrated full-motion video, high-speed data, and voice services.

Frame relay service is a spin-off of the work done on ISDN and uses a simplified version of the $X .25$ protocol, thereby achieving the much higher transmission rate of up to 1.5 megabits per second. Switched multi-megabit data service (SMDS) uses the existing T1 (1.5 megabits per second) and T3 (45 megabits per second) facilities and some implementation of ATM protocols. In short, frame relay is the next generation of X.25 networks, and SMDS is the next generation of leased T1 and T3 circuits. Both networks represent a change to switched-bandwidth, on-demand service.

There are many technologies that are not presented here, the most notable being FDDI-II, a follow-on to FDDI that allows voice, video and many of the WAN services. While it is on the drawing board, it may never make it into a production environment if ATM lives up to its promise.

\subsection{Applicability}

Its 64-kilobit-per-second-per-channel data rate makes ISDN a candidate for replacement of RS-232 serial lines. However, some experts see ISDN as a limited technology, too slow for workstations and high-end PCs and now overshadowed by faster and bettersupported technologies.

Frame relay is an advanced switching technology that statistically multiplexes data packets over a carrier such as a 56-kilobit-per-second line, or over T1 (1.5 megabit-per-second) lines. This technology uses time division multiplexers (TDMs) to keep per-packet overhead low. Frame relay networks are used in commercial applications where cost is a large factor and the low data rates are acceptable. The telcos also support frame relay for use in WANs. Good vendor support is already available for frame relay, with more than 30 vendors supplying equipment.

\subsection{Trends and Timeframes}

Introduction of ISDN in the United States met with some difficulties because of the breakup of AT\&T in 1984 and the lack of a public telecommunications policy. Despite these difficulties, the deployment of ISDN in the United States is proceeding. By the end of 1992 about 1000 telephone company switches (out of 15,000 total) had ISDN service. The deployment will have reached 60 to 80 percent of all 
Frame relay is not without its share of problems.
Video conferencing is in widespread use on private networks and on ESNET. lines by the end of 1994. The introduction of the National ISDN Program, initiated by the Bell Operating Companies and Bellcore and scheduled for the end of 1992, should help achieve this milestone.

Because of significant differences in their telecommunication environments, deployment of ISDN in Europe, Japan, Singapore, Hong Kong and Australia is far ahead of the United States.

We can expect the telcos to aggressively market ISDN. It will remain the fundamental infrastructure for digital networks well into the 21st century, serving millions of individual and business customers.

Work on the next phase of ISDN implementation, Broadband ISDN (BISDN), began in the mid-1980's and moved forward fairly rapidly, owing to major developments in optical networks (SONET) and fast packet switching using ATM.

Central-office-based ATM switches will be available sometime between 1995 and 2000. BISDN services will be available to the public after the year 2000 .

\subsection{Technical and Management Issues}

Frame relay is not without its share of problems. Most notably, it is hampered by connectivity problems between local telephone carriers. Because of its low speed, frame relay is not used in scientific data communications and may never be used in the DOE complex except for WAN communications.

\section{Video Conferencing}

High-speed networking has brought many new tools to bear on the problems of collaboration among geographically dispersed coworkers. In general, these tools support what is coming to be called computer-supported cooperative work (CSCW).

The software for CSCW is known as groupware. Early forms of groupware include e-mail, teleconferencing, and facsimile transmission. Video conferencing is yet another form of groupware, one now in widespread use on private networks and on ESNET.

Video conferencing gives a group at one site the ability to see and talk with groups at one or more other sites. TV cameras at each site are used to view the group and to display visual material such as slides. TV monitors display the images at and from each of the remote sites. 
Video applications on the workstation will reduce the overhead in setting up video conferences.
To reduce the network bandwidth requirements, video conferencing systems employ some form of data compression.

In addition to providing the basic video service, commercial systems can capture and manage slides, send and receive facsimiles, record meetings on videotape, and play the tapes to remote sites. Video switches provide intelligent management of conferences involving three or more sites so that all viewers can see the speaker simultaneously.

Although video conferencing currently depends on commercial systems located in a conference-room environment, it is also possible to capture and send video and sound from a conventional workstation and to receive and present the same video and sound at another (see Section 11).

\subsection{Applicability}

Because of the increasing size and geographical extent of scientific collaborations, it is critical to develop and use technologies that improve communication among the collaborators. Video conferencing has already proved its usefulness in allowing more frequent contact than would be possible if it were necessary to travel to meetings. The development of video applications on the workstation will improve communication further by reducing the overhead in setting up video conferences.

\subsection{Trends and Timeframes}

Video conferencing has been available for many years and has been used in business and in the DOE Defense Programs. However, early commercial systems were expensive, and were not widely installed. In addition, they required line speeds of at least 768 kilobits per second, more than was available to most sites. Commercial video conferencing has therefore been used little in DOE programs.

When less expensive systems were developed that offered multisite capability and used lower speeds, they were installed in many of the DOE labs. In early 1990, DOE's Office of Energy Research began a pilot program running over ESNET. The program used dedicated bandwidth of 384 kilobits per second between LBL, Fermilab and the SSC Lab. Recently, the pilot program has grown to include other DOE labs and international sites. During the two-year program, enhancements to the compression software have provided better quality at lower speeds. The links now operate at 128 kilobits per 
Workstation-based video conferencing is beginning to appear in prototype implementations.

Video conferencing results in increased network load. second. This pilot has proved so useful that the program is being expanded to include more sites, and ESNET management is developing a plan to support its needs. This support will include both dedicated links and bandwidth-on-demand.

At the same time, workstation-based video conferencing is beginning to appear in prototype implementations (see Section 11, Desktop Communications). The earliest versions use conventional compression/decompression (CODEC) systems to capture the video and sound. The output of the CODEC is then processed by the workstation. This processing wraps the output in IP packets for transmission over the network. Workstations at the receiving end then unwrap the packets and decompress the video and sound for presentation on the workstation.

\subsection{Technical and Management Issues}

Although there is an emerging communication standard for video conferencing (the H.261 standard), each of the vendors has additional features that this standard does not support. This means that interoperability between different systems results in a loss of functionality. The introduction of workstation-based systems may create even more variants in the standards.

Video conferencing results in increased network load. With the current system of dedicated bandwidth, in which a video conference would have exclusive use of a network channel, the number of possible links must be tightly controlled. Even with packet-based systems, however, the demands on bandwidth will grow, and video conferencing applications will require immediate response to ensure that the video and audio streams are not interrupted. This will require modifications of network priority systems.

Finally, multisite video conferencing requires IP-multicast, using packet video. Otherwise, each link in the conference would have to carry multiple copies of the conference, one bound for each remote site. IP-multicast addresses this by sending only one copy over each link and then splitting it at routers for transmittal to the separate sites. The multicast protocol must be incorporated into the network routers.

\section{Desktop Communications}

As video conferencing becomes increasingly integrated with data networks and computers, it will also move from the conference room 
During the next two years workstation-based video conferencing will move from prototype to production. to the desktop environment. In one recent example, researchers used workstation audio and video to conduct a working conference over the The Internet and ESNET in which a dozen "attendees" on remote workstations had full interactive audio-video capability and hundreds of passive workstation participants on several continents were able to view and hear the proceedings.

Continuing innovations, such as the merging of the telephone handset, the desktop computer, and digital video technology, will provide new office video conferencing capabilities that promise increased productivity for the information worker.

\subsection{Applicability}

Any worker who handles information or needs to communicate with distant associates will benefit from the ability to share an array of audio and visual media in a conference format. The coming availability of greatly improved resolution for computer-based graphics will make transmission of graphic information more feasible for such purposes as long-distance collaboration on reports and presentations, and viewing at videoconferences. This multimedia dialogue will lead to faster decision-making and broader participation in ad hoc meetings within and across organizations.

\subsection{Trends and Timeframes}

During the next two years workstation-based video conferencing will move from prototype to production. Tools such as shared whiteboards will augment conventional video conferencing, pernutting exchange of drawings and other documents. Improved video capture and compression systems for workstations will make the comnercial CODECs unnecessary. Video conferencing will be as easy and as common as e-mail is today.

The H.261 standard addresses video CODEC equipment standards for sending and receiving video signals in a compressed format to save bandwidth. This standard allows for chip-based CODECs, which will promote compatibility among the various vendors' implementations and thereby reduce the cost of workstation and PC-based video. The H.261 standard also allows interconnection of existing video systems, but does not address higher resolution video or multimedia applications. Users are therefore likely to continue selecting videoconferencing equipment on the basis of proprietary protocols for several years yet. 
Video phone systems are, at present, mutually incompatible and amount to nothing more than video telephone calls. Workstation and PC video systems are also usually not interoperable but can greatly extend the function and usefulness of video phone systems, at a cost of about $\$ 2100$ per seat. Recent video phone announcements by long haul carriers are an indication that more desktop video innovations are on the way.

Within five years, video conferencing will establish itself in the office and even on the desktop, albeit in a proprietary form. Within a few years, digital data protocols will be able to handle multipoint conferencing at speeds lower than 64 kilobits per second per path. This capability has already been demonstrated in the video conference referred to at the beginning of this section.

The technologies for chip-based CODECs, audio/video desktop equipment, and transmission protocol will continue to merge with each other, allowing coworkers in a teleconference to work jointly on spreadsheets or graphics and cooperate in other sophisticated information analysis activities.

\subsection{Technical and Management Issues}

Investments in proprietary systems should be limited.

Data acquisition is a major component of computing systems used for experiments.
Information managers and telecommunications and computer center managers should begin acquiring or testing desktop communications systems within the next two years. However, to avoid a proliferation of incompatible systems and premature obsolescence, early acquisitions should allow for technological evolution and expansion, and investments in proprietary systems should be limited.

\section{Data Acquisition and Control}

Data acquisition and control, or "real-time systems," consist of the specialized hardware and conventional computers that are used to control experimental apparatus and to record data for future analysis or signal processing. These systems are frequently as simple as tape controllers coupled to analog-to-digital converters. However, as experiments have become more complex, more sophisticated data acquisition has become necessary to cope with larger data samples and to filter and process data before they are recorded. Data acquisition has become a major component of computing systems used for experiments. 
An important aspect of the data acquisition problem is image capture and processing.
Data acquisition systems are becoming a key feature of the heterogeneous distributed environment.

\subsection{Applicability}

Data acquisition and control is critical to almost all areas of the DOE research program. The most demanding application:s are probably still in High-Energy Physics and Nuclear Physics, whose large detector systems can generate from 1 to 10 megabytes of raw data per second. (Detectors planned for the Superconducting Super Collider are expected to increase this rate to 100 megabytes per second.) Because experiments conducted in these programs typically run for many months at a time, the total data load is between 1 and 10 terabytes per year and will grow to a petabyte per year by the time the SSC begins operation. Other areas of the DOE program have generally similar requirements, but differ in data rate, duty cycle or types of processing.

An increasingly important aspect of the data acquisition problem is image capture and processing. Facilities such as the electron microscopes and synchrotron light sources will use imaging techniques to explore a wide range of phenomena in chemistry, material sciences and life sciences. For these research programs it is important to store and manipulate images as well as to render and display them, frequently in real time while the experiments are running. This real-time processing requires coupling of storage systems with high-performance computers.

\subsection{Trends and Timeframes}

Traditional data acquisition and control systems were developed as special appiications and did not, for the most part, employ commercial networking or computing hardware. In the case of high bandwidth detectors the performance of commercial systems was not adequate. Also, hardware standards for data acquisition and control were different from those for commercial computing and networking.

However, within the past few years the performance of commercial networking has improved to the point that these systems can be expected to satisfy most data acquisition requirements. At the same time, modern detector systems are, increasingly, built with embedded commercial processors that can communicate with conventional network protocols. As a result, there is a trend toward moving data acquisition systems out of the area of special purpose systems and instead making them a key feature of the heterogeneous distributed environment. This trend is likely to accelerate so that, in the future, 
The scientists who access samples of data, or control the apparatus, may be located at remote sites. vendors of detector systems will depend even more on commercial computing standards.

\subsection{Technical and Management Issues}

As more conventional computing systems and networking come into use for data acquisition and control, the main technical issues are, in many respects, the same as those for other heterogeneous distributed systems. The most challenging, perhaps, is the development of software systems that manage the flow and processing of large data samples from the detectors. This software must integrate data filtering and compression, database systems, mass storage and visualization.

The scientists who access samples of data, or control the apparatus, may be located at remote sites throughout the world. This presents special challenges for security and data integrity. The quality of the experimental data and even the safety of the apparatus will depend on adequate control of access to the detector.

\section{Software Development Tools}

Development of standard, interoperable software development tools is in its infancy. Many platforms lack a full set of software development tools, and, furthermore, the different software technologies do not exchange data or information. Software development tools fall into the following major categories:

- Traditional tools

- Computer assisted software engineering (CASE)

- Software project management programs (e.g., PERT)

- Automated testing environments

- Cross-platform development environments

- Reengineering tools 


\subsection{Applicability}

Instead of batchprocessing, it is now often preferable to change one or two lines of code and test them immediately.

All current CASE tools have implemented the ANSI waterfall model.

Rapid prototyping is an iterative modeling process.
The traditional software development tools, including compilers, word processors, online documentation, and flow-charting programs, have become increasingly sophisticated. Integrated development environments and incremental compilers are drastically changing the way that programmers work. For example, instead of batchprocessing, compiling, and running large amounts of code, it is now often preferable to change one or two lines of code and test them immediately. This greatly simplifies such problems as comparing the performance of alternative algorithms. The effectiveness of these tools, however is limited to implementing specific software systems in specific operating systems and hardware environments.

Traditional tools and CASE tools have largely evolved in the context of business systems, written in COBOL, where the nature of the work to be done is generally well-defined. CASE tools in particular are often poorly suited to a research environment, where procedures needed to carry out the research may have yet to be discovered. All current CASE tools have implemented the ANSI waterfall model, in which the software development cycle follows a relatively uniform progression, consisting of an isolated phase for definition of requirements followed by distinct phases for design, coding, testing and integration. Assumptions made at the beginning of the development process tend to be written in stone, and changing them later is cumbersome. Except in the case of certain well-defined problems, the benefits of CASE tools are not worth the burden of adapting the development model to follow the ANSI waterfall model.

By contrast, rapid prototyping is an iterative modeling process. First, users and developers discuss preliminary requirements. The developers then produce a prototype - often only a general look and feel interface with no internal working parts. The users and developers review the prototype and produce a new set of requirements. This cycle is repeated until the users are satisfied or a cutoff point is reached. Rapid prototyping is often the optimal software development methodology for DOE research programs, and is the only available methodology for "wicked problems," commonly encountered in research, in which neither the exact nature nor the solution of the problem is known. Unfortunately, except for a few flow-charting utilities and the traditional tools, CASE tools do not support this methodology. However, some newer development methods were created with this methodology in mind. 
Object-oriented

programming

accommodates rapid

prototyping.

Tools for automated

testing are improving rapidly.
DOE programs will

gain little from

traditional CASE in the near future.
One menodology that accommodates rapid prototyping is objectoriented programming (OOP), which is emerging as an alternative to traditional CASE strategies. Unlike most CASE methods, OOP uses a recursive development process that is tolerant of design changes. OOP conceives of software programs as cooperative collections of objects, rather than as a hierarchy of subroutines. This objectorientation brings with it the potential for improved software reusability and interoperability. In short, OOP is more flexible than traditional CASE for scientific and technical computing.

An area in which CASE tools have proven useful is relational database design. For instance, design tools for relational databases integrate the drawing of entities (the entity relationship diagram) with the definition of the entity's attributes (the data dictionary). They :an also produce the database language constructs needed to create the database objects and provide queries to them. These tools still cannot produce actual modules, but can assist in rapid prototyping.

Further along in the software development life cycle, tools for automated testing are improving rapidly. Automated testing involves use of a master computer to send instructions to a slave computer, which then executes the program to be tested. The master computer can recover from a crash of the slave computer, flag the error and restart the testing process.

Interface builders that automatically generate code are becoming increasingly convenient and powerful. These tools can drastically shorten development time by allowing an interface designed on one platform to be immediately ported to other platforms. Especially atiractive are cross-platform interface development environments, which now support a number of user interface designs. The interface for each platforiıi appears just as a user of that platform would expect. However, these tools only implement cross-platform interfaces, leaving unaddressed many machine dependencies.

\subsection{Trends and Timeframes}

Traditional CASE is still a growth industry. Since most software development worldwide is still in COBOL and targeted for financial instizutions, this trend will probably continue. For the most part, DOE programs will gain little from traditional CASE in the near future. 
The populnrity of $O O P$ will prubably increase over the next five years.
Within the CASE community itself, the debate continues as to whether CASE and OOP are compatible or are competitors. Except for databases, where one-hundred-percent automatic machine code and $\mathrm{COBOL}$ generation from high-level, fourth-generation language (4GL) constructs are now possible, CASE has generally proven inadequate. Many developers use some of the flowcharting capabilities, but most CASE packages are mothballed. This is not likely to change so long as CASE is based on the waterfall model.

The popularity of OOP will probably increase over the next five years as a new generation of programmers is introduced to its capabilities and as machines become faster, making OOP's inherent inefficiencies insignificant.

Cross-platform development tools will eventually develop into a virtual machine programming environment, with file I/O models, interface design models, and memory management models so that code will be truly portable across platforms. Early benefits from this technology can be expected in 1993.

Finally, here are quick projections for the other technologies mentioned:

- Given current technologies, software reengineering is an intractable problem and will not become possible for many years, if ever.

- Automated testing is currently very useful and will continue to improve.

- Long-distance computer-mediated collaboration will be developed, but only as stand-alone products.

\subsection{Technical and Management Issues}

Training is the primary management issue.
Training is the primary management issue. Allowance must be made for programmers to come up to speed on new development environments.

Experimental computer systems are not likely to have any sophisticated development tools, perhaps not even high-level compilers. Management's awareness of these hardware constraints is important in projecting completion dates.

If software development services are to be integrated at the user's desktop, the ability of these services to exchange data and control 
information must improve significantly. Otherwise, a user might have to contend with, for example, FORTRAN development, concurrent engineering CAD/CIM, and desktop video conferencing environments that share hardware and network infrastructure but cannot exchange data productively. A critical research and development issue, then, is the development of standards to promote data exchange between environments.

\section{Scientific Database Management Systems}

Scientific data have typically been managed using specialized software and ordinary sequential files.
The relational model is inadequate for scientific databases.
Database requirements for scientific applications differ significantly from those for business applications. In general, scientific databases are characterized by complex data structures and operations, neither of which has been well provided for in commercially available database management systems (DBMSs). As a result, scientific data have typically been managed using specialized software and ordinary sequential files. Only recently have database technology and the performance of commercial database products improved to the point that their use for scientific applications can be considered. The following are basic functional requirements for both scientific and commercial DBMSs:

- Data models that use abstractions to represent the structure and semantics of the data.

- High-level query languages for accessing and manipulating the data.

- Support for concurrent access by multiple users.

- Mechanisms for expressing and validating the integrity of the data.

- Backup and recovery facilities.

- Support for efficient storage on and access from secondary storage.

Commercial database technology, now almost universally based on the relational model, has successfully implemented these capabilities for business applications, but not, so far, for scientific applications.

The main modeling capability of the relational model consists of multiple tables that can be linked by associating columns. This simple model is inadequate for scientific databases. Many types of scientific 
Commercial DBMSs

have serious limitations in their ability to maintain the integrity of data.

New mechanisms are needed to support long transactions. data cannot be organized into tables as required by the model. For example, ordered structures such as DNA sequences or temporal sequences cannot be represented directly. Other data types such as vectors and matrices also cannot be easily represented. Furthermore, the order of the rows in a table, which correspond to instances, is not enforced in relational databases. Thus, an ordered sequence cannot be represented simply as a sequence of entries in a relational table. In addition, scientific analysis by its nature requires a more powerful query language-capable of formulating more complex queries-than structured query language (SQL), which is the de facto standard of commercial relational database systems.

The need to control concurrent access to data-to prevent update transactions from interfering with one another-is vital for business systems such as banking, accounting, or reservation systems. While concurrent access is not an important issue for scientific databases, there is a need to support multiple versions of datasets and to keep track of the correspondence between them.

Commercial DBMSs have serious limitations in their ability to maintain the integrity of data. Even a simple feature that checks that data values are only from a given list of "categorical values," often needed in scientific databases, is not always supported. One of the main problems in relational DBMSs is making sure that data values in one table are synchronized with values in other tables, so that, for instance, a person's identifier appears in all the tables that contain information about that person. This feature, called "referential integrity," is still not supported automatically by commercial systems. Integrity becomes more difficult to maintain when multiple domains are involved, as in checking that a person's birthdate is smaller by at least 18 years than the employment start date. In general, integrity conditions may require the power of a programming language for their expression.

If transactions are short, as they frequently are in business databases, it is acceptable to back up to the last successful transaction for the purpose of recovery. For scientific databases, where long transactions are the rule, too much work may be lost. New mechanisms are needed to support long transactions. 
Physical storage of data according to their spatial locality reduces I/O from secondary storage.

\section{Current commercial} database systems support only secondary storage (disk) systems.
Physical data structures and access methods are the key to efficient support of queries. Relational database implementations typically organize the rows in tables as records in files, and provide indexes over such records. The access requirements of scientific applications, however, favor other ways of clustering data. For example, spatial applications typically use local access operators, such as "find neighboring points" for calculations of mesh data. In such a case, physical storage of data according to their spatial locality reduces the amount of $\mathrm{I} / \mathrm{O}$ from secondary storage.

\subsection{Applicability}

The use of more sophisticated data collection devices that generate more data, and the relatively low cost of computing components that process experimental data or generate data from models, are resulting in databases of increasing size. Consequently, scientific databases pose a new challenge, that of efficient management of information on tertiary storage systems such as tape robots and optical media. Current commercial database systems support only secondary storage (disk) systems, which are too expensive for very large databases.

Another challenge posed by scientific applications is their everbroadening variety of data types and structures. The following are some current and future applications within the DOE program:

- Health surveillance data and health records are now used to track exposures of workers to hazardous materials and to assess the long-term health implications.

- The image data accumulated by researchers in the human genome project are organized and manipulated in a database in which the images function as icons for the selection of appropriate data samples.

- A pilot project organizes data from high-energy and nuclear physics detectors into large databases, using "event type" as a database key. This project has demonstrated that commercial databases can be used for analysis of data from these detectors.

- Database management systems may soon be used to manage complex multimedia data sets, such as video streams from cameras or experimental apparatus. 


\subsection{Trends and Timeframes}

As the commercial

database systems improve, tools for data modeling and schema definition also need to be refined.

New commercial products based on object-oriented technology may allow higher performance levels for large databases.

The extended relational model...

Extensible database systems...
The major successes of commercial relational database systems have been in the business sector, and vendors are only beginning to address the technical issues that have limited the usefulness of these systems for scientific applications. As the commercial database systems improve, tools for data modeling and schema definition also need to be refined in both the laboratory environment and in commercial systems. When this happens, use of commercial databases will increase significantly.

The recent appearance of new commercial products based on objectoriented database technology, an approach that seems better-suited to scientific data, may lead to higher performance levels for large databases. An object-oriented DBMS (OODBMS) combines conventional database techniques and the object programming paradigm (see Section 13, Software Development).

Two main features, emcapsulation and inheritance, make the OODBMS attractive for use with large, complex datasets.

Encapsulation is the ability to define the data structures and operators associated with an object and make them available at the user interface, while hiding the details of how the data structures and operations are implemented. Inheritance allows subclasses of objects to retain properties (inheritance structures) that they hold in common with their parent class. This allows objects to share code.

Another possible approach for scientific databases is to extend a popular database model by adding inheritance structures and new data types such as vectors, matrices, images, and even procedures. This approach has lead to several prototype systems based on an extended relational model.

Finally, a third approach, called "extensible database systems," advocates the creation of a customized data management system for a given application. These systems use building blocks of reusable software that the user can select according to need. The user can design additional blocks as necessary. The key to this approach is the design of the interfaces (the glue) that permit the interchangeability of modules and the integration of new modules. One problem is that the user who designs such a customized system must himself be fairly expert in the implementation of DBMSs, and this approach may be more appropriate for a software house that designs custom DBMSs. 
The characteristics of each application must be well understood before a model can be developed.

Current technology does not readily support heterogeneous database systems.

\subsection{Technical and Management Issues}

The three types of systems discussed, OODBMSs, extended relational systems, and extensible database systems, all support user-defined data structures and operations. However, each of these approaches has different strong points. Future research should concentrate on evaluating such systems with real scientific applications. This may lead to the development of systems that incorporate some of the important advantages of the three approaches.

The characteristics of each application must be well understood before a model can be developed. In addition, new methods for supporting the application may need to be developed. To be successful, such developments require a detailed understanding of the discipline as well as of the needs of the scientists in those disciplines. Because of the diversity of scientific applications specialized interfaces may need to be developed even if a common underlying DBMS is used. Such customization is crucial in order for scientists to communicate in symbols, jargon, and graphical or imaging representations peculiar to their application. Thus, tools for quickly developing customized interfaces are also important.

In selecting a DBMS, one must also bear in mind that current technology does not readily support heterogeneous database systems. From a management perspective it is very difficult, in practice, to agree or impose standard DBMSs on communities of users. One way to mitigate this problem is for experiments or disciplines to settle on interchange format standards. Several such standards are already in use. It will be necessary for scientific DBMSs to be able to load such standard data formats, as well as produce them.

Finally, it is often the case that commercial products are too expensive for small research groups, making it difficult for them to share data from more well-financed collaborators. Thus, a management strategy should include plans to take advantage of electronic communication, which is rapidly growing in terms of capacity and reachability.

\section{Scientific Visualization and Virtual Reality Tools}

Scientific visualization is a further development of traditional CRTbased computer graphics work, made possible by improvements in the underlying computer hardware and software. Virtual reality is a three-dimensional graphical environment. Using special I/O devices such as a visual headset and data gloves, it reduces the boundary 
between the computer and human perception to a transparent user interface.

\subsection{Applicability}

Visualization technology is essential in understanding the complex data that results from large-scale scientific computations. Formerly, custom-written graphics systems were used, but these are being replaced by Application Visualization System (AVS) and AVS-like graphics environments. These programs can run on most high-end color workstations, and include a software migration path from the workstation to a graphics supercomputer. For example, AVS is being used on workstations to "visualize" the behavior of complex fluid flow from data computed on a Cray supercomputer, and SGI graphics workstations have been connected to computational resources via high-speed links to allow interactive investigation of computational fluid dynamics (CFD) data computed on a topology of 512 by 512 by 512 points.

Virtual reality is still in its earliest stages of development, but it has enormous potential. For example, a worker could use virtual reality tools to operate a robotic alter ego via remote control in a damaged reactor core. Data gloves and a visual headset would connect the worker electronically to the "hands" and "eyes" of the robot, which might be hundreds of miles away.

\subsection{Trends and Timeframes}

During the past few years a number of visualization software packages have appeared.
During the past few years a number of visualization software packages have appeared-including AVS, Data Explorer, Explore, and $\mathrm{aPe}$-that couple a graphical programming environment with data exploration modules. The user can then combine graphics and data analysis modules into a "network" that implements a set of data transformations and graphics algorithms, and displays the results. Using these tools, the user can interactively explore and display the data without having to write a custom program.

Newer high-performance graphics workstations, such ais Silicon Graphics Crimson and Stardent Titan, show continued improvement in performance and decreasing cost. These workstations generally have been designed and optimized for computer graphics and contain special hardware for accelerating graphics functions. In general, they can be used by the newer visualization software and have replaced mainframes as the preferred platform for computer graphics. 
It is now a standard practice on massively parallel computers to use framebuffers attached to high-speed I/O channels, such as standard HIPPI channels, to expand the capability and increase the reliability of existing software and hardware. Thinking Machines Corporation was among the first to use this technique for real-time display of images generated on a massively parallel processor.

Finally, there has been significant progress in developing highresolution video projectors to project images on large-format screens. It is now common practice to project $1280 \times 1024$-pixel 24-bit color images on 10-foot screens. Future systems will have higher resolution and lower cost.

Various user interfaces have been developed for virtual reality systems. In one, the user wears a stereoscopic head-mounted display with small CRTs positioned in front of each eye. A small motion tracker attaches to the bridge of the nose, and the user wears special data gloves that contain motion sensors. The computer projects a 3D image of a graphical environment, and the user is free to interact with this environment, using the data gloves to grasp or push objects (causing a change in the display) and turning or tilting his or her head to look at a different perspective of the simulated environment. The user can also interact with the displayed data in ways not possible in the physical world. For example, the user can "fly" or "swim" through the space.

Another approach uses coordinated multiple video projectors to beam a 3-D image on the sides of a cube made of rear-projection material, providing a 3-D virtual world for more than one viewer.

As more applications are found for this technology during the next 3 to 5 years, we should see dramatic improvements in user interface devices such as the head-mounted displays, data gloves, virtual reality rooms (such as CAVE and Holodeck), force-feedback interfaces, motion trackers, audio projection, and sensory immersion. Currently, a low-end demonstration system can be assembled for less than $\$ 100,000$, including the computer hardware needed to drive the graphics. We expect this cost to decline rapidly over the next decade. 


\subsection{Technical and Management Issues}

It is not feasible to give each researcher a highperformance graphics computing capability.

A variety of systems should be made available with which users may experiment.
As scientific visualization hardware and software become critical components of the computational science environment, integrating this technology into the existing computing infrastructure becomes a problem. It is not feasible to give each researcher a high-performance graphics computing capability in addition to a computer workstation and other necessities. One possible approach is development of compatible software tools that run on both the user's workstation and the high-end graphics computer; tools such as AVS can run on both relatively low-power workstations and high-end platforms. The user would then be able to prototype visualization schemes, using the fast graphics machine only after most of the bugs had been worked out. The large I/O requirements of graphics machines used for scientific visualization often mean that networks must be improved or upgraded from Ethernet to FDDI or HIPPI/FCS to support the data requirements.

This field is opening up quickly, and systems are on a rapid development cycle. As a result, there are few, if any, effective standards. The standards that do exist are defacto standards based on tools that have become successful in the market. Because standards are lacking and the technology is changing rapidly, this emerging technology deserves thoughtful evaluation, and a variety of systems should be made available with which users may experiment.

\section{Hypermedia}

A conventional paper document consists basically of text, figures, tables, and, possibly, references to other works. Using a workstation, one can add video and sound, creating a multimedia document that doesn't have a paper analog, but still organizes the material in a serial fashion.

A hyperdocument departs from the serial organization of a multimedia document. It includes links that permit the reader to follow various routes through the document, depending on such factors as level of expertise and interest in the material.

In addition to giving the user the ability to explore the information space, hypermedia also allows the user to customize a document by adding links and annotations. 


\subsection{Applicability}

Hypermedia will impact

how scientific

information is conveyed

to other scientists, to

students, and to

industry.
Commercial products are available that will assist creators of hypermedia.
Hypermedia will have a significant impact on how scientific information is conveyed to other scientists, to students, and to industry. The creator of a scientific hyperdocument can provide suggested paths through a document, depending on the reader's sophistication or need. Some hypertext programs include an overview function that displays the hyperdocument's network structure. Reference material can be linked with the hyperdocument to assist the reader in following the development of an idea. Figures or multimedia linked to the hyperdocument can provide vivid displays of results or apparatus.

For documents that describe computation, the hyperdocument can use all the power of scientific visualization or virtual reality.

Mathematical expressions can be accompanied by computer code that evaluates the expressions and displays the results. With the help of visualization tools the user will be able to explore large databases or spreadsheets. Other uses will include public information and safety documentation.

\subsection{Trends and Timeframes}

An increasing number of commercial products are becoming available that will assist creators of hypermedia. Products for the PC include utilities for integrating sound and video with electronic annotation. Products based on the Standard Generalized Markup Language (SGML) and other standards are also emerging. The next few years will see an enormous growth in the number and quality of these products, accompanied by a complete change in the way that science or other complex information is presented.

\subsection{Technical and Management Issues}

The printed document has the advantage of requiring no special equipment or software tools. The simple exchange of electronic text is also relatively easy, because all workstations can handle ASCII characters. However, at any level of sophistication beyond plain text, electronic documents require significant effort. Document preparation using $T_{E X}$ or other document formatting programs requires agreement among users on macros and other softwarespecific functionality. Figures, tables and formulae also depend on specialized software or standards. Hypermedia will require new software at both the "author" and "reader" sites. Unless standards 
Networks and peripherals used to access hyperdocuments may need to be upgraded.
Computational chemistry...

Structural biology... are adopted, each site will need copies of all programs in use in the community.

The size of hyperdocuments means that, usually, they will be impossible to deliver on floppy disks. The obvious alternatives are CD-ROMs or similar large-volume storage devices, or using a highspeed network. There are advantages and disadvantages to each in terms of performance and ease of use. It is likely that both portable storage media and networks will be used, depending on the application. By the same token, networks and peripherals used to access hyperdocuments may need to be upgraded.

\section{Challenges in High Performance Computing}

The DOE is one of four major Federal agencies involved in the High Performance Computing and Communications (HPCC) Initiative. The goals of the Initiative are to extend the country's technological leadership in high performance computing and commurications, to hasten innovation in the information technologies in service of national education and security needs, and to enhance the nation's industrial competitiveness.

\subsection{Applicability}

The DOE has identified a total of six Grand Challenge projects to be undertaken at its High Performance Computing Research Centers (HPCRCs). The projects discussed in this section illustrate the types of studies now in progress.

The Computational Chemistry project is a study of critical problems in (1) incineration of halohydrocarbons (major causes of contamination at many of the nation's waste sites) and the development of replacements for chlorofluorocarbons, which have been implicated in the destruction of the ozone layer in the upper atmosphere; (2) sorption and reactions of pollutants on and in clay minerals, which are processes important in the remediation of contaminated soils and groundwater; and (3) the rational redesign of biodegradative enzymes to enhance the effectiveness of bioremediation of polluted soils and groundwater.

The Structural Biology project studies crucial problems in (1) protein folding (structure) and protein-protein interactions, and (2) detailed DNA sequence analysis and interpretation. The first of these is the key to all body functions-growth, digestion, healing, fighting 
Petroleum Reservoir and Groundwater Modeling...

Numerical Tokamak...

The Grand Challenges are a test bed for collaborative projects in distributed computing. disease, storing and releasing energy, and degradation. DNA sequence analysis and interpretation is the key to understanding hereditary characteristics, including diseases and susceptibility to disease.

The Petroleum Reservoir and Groundwater Modeling project will enable more effective studies of two very important national problems: groundwater pollution migration/remediation and enhanced oil reservoir recovery. The object of this project is to develop algorithms for modeling flow through porous media that are suitable for the new high pertormance massively parallel computer systems.

The tokamak design for a thermonuclear fusion energy source is on the verge of demonstrating the long-sought goal of scientific "breakeven"-producing as much energy as it takes to run the experiment. The Numerical Tokamak project will play a profoundly important role in successfully developing the tokamak as a commercial source of electrical power.

These Grand Challenge problems involve fundamental science and engineering issues with broad scientific and economic impact, but require unprecedented computational power for solution. The Grand Challenges also function as a test bed for collaborative projects in distributed computing and further the DOE objectives of forming collaborative ventures with universities and industry; nine national laboratories, 16 universities and more than 40 businesses will participate in or fund the projects.

Although the research teams involved in the six projects are spread across the nation, they will use the National Research and Educational Network (NREN) to communicate with each other and with the HPCRCs at Los Alamos National Laboratory and Oak Ridge National Laboratory. The HPCRCs will provide highly advanced applications research and technology development to permit rapid progress on the Grand Challenges.

In addition to the Grand Challenge problems, there are many other problems of comparable complexity. Examples of other DOE projects on this level include Global Climate Modeling (GCM) and Materials Technology.

The GCM project is a well-integrated initiative that involves research and model development. It is sponsored by the DOE HPCC Initiative 
Materials technology...

So far, most of the computers being marketed are either for a single user or a few users. and the Computer Hardware, Advanced Mathematics, and Model Physics (CHAMMP) program. The GCM project is part of a national effort to develop complete global climate models that couple atmospheric, oceanic, sea ice, and land surface processes. Until now, available computational power has been insufficient to permit models that encompass ail these layers.

Materials technology is critical for US industry and defense. The new materials of greatest interest have such complex properties that their process development and characterization are limited by conventional methods. The development of new, parallel algorithms in this field will also influence future computational trends.

\subsection{Technical and Management Issues}

Many issues, of which some are new and some have already been addressed, confront our efforts to use massively parallel computers to solve these problems. Special expertise is needed to program these machines, and there are also issues of accurate measurement of machine performance and finding debugging techniques to assure correct results.

So far, most of the computers being marketed are either for a single user or a few users. These resources must, then, be allocated with care if they are to be cost-effective. Problems of charging for usage must also be addressed, as well as the enormous networking issue. To balance the very high computational potential and the trend toward distributed computing, gigabit-speed networks must be in place, not only locally, but for applications distributed across the country.

\section{Computing Technology in Agile Manufacturing}

Agile manufacturing involves the electronic integration of design and manufacture. Using a distributed computing system on a factory LAN, agile manufacturing links designers and analysts on workstations with manufacturing equipment on the factory floor. Its use can greatly reduce manufacturing costs and flow-time. One area in which agile manufacturing has been implemented commercially is stereolithography.

Stereolithography is a manufacturing process that uses a laser beam to draw an image on the surface of an organic liquid known as a photopolymer. The surface hardens into a very thin film of solid plastic wherever the energized laser beam strikes it, but remains 
The factory's LAN is used as an "electronic production line." liquid elsewhere. The film innage is lowered on an elevator just beneath the liquid's surface and the process is repeated many times, building up a stack of thousands of thin layers of polymer to form a solid laminate. When the laser beam's motion and intensity are programmed to match a 3-dimensional computer model of a surface or object, the resulting plastic laminate is an exact replica of the part designed in the computer.

The factory's LAN is used as an "electronic production line." The file containing the part's data model is the "electronic workpiece." The designer designs the part using a graphics workstation and passes the part's file to a mathematician at another workstation. There, the database is checked for consistency, correct geometry and topology. Next, the "electronic part" file is given to a numerical analyst who adds any geometry necessary to support the part during fabrication. The datafile is then "sliced" into the thin layers that the stereolithography laser wili trace out to form the product. Finally, the set of slices is transmitted through the network to the stereolithography machine in much the same fashion that a plot or printer file is sent 10 its output peripheral. All this takes place without the exchange of any paper drawings or blueprints among the manufacturing team participants.

\subsection{Applicability}

Stereolithography was developed in the late 1980 s and is becoming common in industry. It is used for design of industrial parts that have complex geometry, such as gears, turbine blades, castings and intricate assemblies.

With cc nventional fabrication methods, mistakes in perception of geometry can be very expensive and time-consuming to fix, because of the tooling and set-up required before the first part is made. With stereolithography, a sample or prototype that matches the shape of the part can be made in a few days at much lower cost.

This combination of information and manufacturing process technologies has recently been applied to biomedical models. Molecular and computational biologists have long performed research with computer models of DNA, proteins, viruses, antibodies and other biomedical structures. These models may be derived from $x$-ray crystallography, or may be computed from first principles of chemistry and physics. 
Stereolithography

models may become

available to the

biomedical research and

educational

communities.
A factory's internal electronic production line will be linked to a wide area network.

A library of standard data model components will be developed.
Modern graphics workstations have allowed researchers to create remarkable displays of these models, but creating tangible solid models has usually been done with "tinker-toy" or ball-and-stick models. For large or complex models, such techniques are extremely time consuming and expensive.

In October 1992 a DOE manufacturing team, working with bioresearch scientists, succeeded in using laboratory data to create a stereolithography model of a portion of a complex antibody structure. DOE's production team used the same electronic production line technique described previously. If such models can be created in a routine production environment, relatively low-cost, high-quality stereolithography models may become available to the biomedical research and educational communities. Such models would be particularly useful in educating high school and college students in structural biology.

Large-scale use of these models will depend on achieving lower manufacturing cost, as through the use of injection molding and investment casting. Current limitations include the inability to color and code parts of the models.

\subsection{Trends and Timeframes}

By 1995, enhancements of manufacturing with electronic production lines and stereolithography can be expected to include the developments described in the paragraphs that follow.

A factory's internal "electronic proàuction line" will be linked to wide area networks such as Internet or a special Human Genome Information Network (HUGIN). This will allow the DOE scientific and research community (as well as the nation's biomedical research facilities) to access production modeling and stereolithography prototyping facilities quickly and easily. The stereolithography capability would simply appear as another network peripheral.

A library of standard data model components or modules in a "stereolithography ready" form will be developed, much like a library of standard programming subroutines. This would allow more rapid turnaround of requests when researchers make only simple changes to a basic model or combine simpler components to make more complex structures. 
Alternative rapid prototyping techniques will be developed.
Alternative rapid prototyping manufacturing techniques will be developed that are compatible with the data models and file structures commonly encountered in biomedical models. These manufacturing processes would offer materials and product features . that may be equal to or superior to today's stereolithography.

\subsection{Technical and Management Issues}

There is a need for an efficient DOE system to administer and prioritize requests to use a future network stereolithography peripheral. DOE scientists are now beginning to work with production engineers on this topic. In addition, cheap massproduction techniques may be needed for a few significant or canonical biomedical models to be used in schools.

\section{Conclusion}

The emphasis in IT development has shifted from technology management to information management, and the tools of information management are increasingly at the disposal of endusers, people who deal with information. Moreover, the interactive capabilities of technologies such as hypertext, scientific visualization, virtual reality, video conferencing, and even database management systems have placed in the hands of users a significant amount of discretion over how these resources will be used.

The emergence of high-performance networks, as well as network operating systems, improved interoperability, and platform independence of applications will eliminate technical barriers to the use of data, increase the power and range of resources that can be used cooperatively, and open up a wealth of possibilities for new applications.

The very scope of these prospects for the immediate future is a problem for the IT planner or administrator. Technology procurement and implementation, integration of new technologies into the existing infrastructure, cost recovery and usage of networks and networked resources, training issues, and security concerns such as data protection and access to experiments are just some of the issues that need to be considered in the emerging IT environment.

As managers we must use technology to improve competitiveness. When procuring new systems, we must take advantage of scalable resources. New resources süch as distributed file systems can 
improve access to and efficiency of existing operating systems. In addition, we must assess opportunities to improve information worker productivity and information management through technologies such as distributed computational visualization and teleseminar applications. As the size of work groups increases, we must provide organizational structures for $\mathrm{CSCW}$, video conferencing, and global data sharing. To enhance DOE's service function, resources such as hypermedia can be used to convey information to science, students, and industry.

In support of its programmatic needs and overall mission, DOE must continue to promote development of new resources such as highspeed networks and infrastructure software, and pursue research in new applications. Such programs as the Grand Challenges, for instance, are testbeds for collaborative distributed computing.

Over the next few years, investment in new technology should be prudent-conservative but forward-looking. It will not be easy to balance short-term needs and long-term strategies. Difficult as these choices are, however, they are necessary if DOE is to fulfill its mission and remain a leader in information technology.

\section{Contributors}

This year's Information Technology Resources Assessment is the result of a collaboration between the Office of Information Resources Management and the Office of Scientific Computing (OSC) in the Office of Energy Research (ER). It was prepared and edited by Stewart Loken, Director of the Information and Computing Sciences Division, Lawrence Berkeley Laboratory. Contributing technical specialists are: Vianna Briscoe of DOE SF (The Integrated Services Digital Network, Video Conferencing), John Cavallini of ER (Introduction, Desktop Communications), Raymond Cline of Sandia National Laboratories (Distributed Computing, Video Conferencing, Hypermedia), Sam Gibson of Lawrence Berkeley Laboratory (The Integrated Services Digital Network), Stan Kluz of Lawrence Livermore National Laboratory (The Integrated Services Digital Network), Stewart Loken of Lawrence Berkeley Laboratory (Data Communication Networks, Data Acquisition and Control, Hypermedia, Video Conferencing, Scientific Database Management Systems), William McCurdy of Lawrence Livermore National Laboratory (Scientific Visualization and Virtual Reality Tools, Hardware for High Performance Computing, Data Storage Systems, Scientific Database Management Systems), Nicholas Nagy of Los 
Alamos National Laboratory (Hardware for High Performance Computing, Challenges in High Performance Computing, Data Communication Networks, Software Development Tools, Data Storage Systems), Arie Shoshani of Lawrence Berkeley Laboratory (Scientific Database Management Systems), Maurice Smith of the Kansas City Division (Computing Technology in Agile Manufacturing, Distributed Computing, Workstations and Administrative Systems), and Rick Stevens of Argonne National Laboratory (Distributed Computing, Scientific Visualization and Virtual Reality Tools, Portable Computing and Communications, Radio Network Technologies).

\section{Glossary}

\subsection{Abbreviations}

$4 \mathrm{GL}$

Fourth-generation language

ATM

Asynchronous transfer mode

AVS

Application Visualization System

BISDN

Broadband ISDN

CASE

Computer aided software engineering

CFD

Computational Fluid Dynamics

CHAMMP

Computer Hardware, Advanced Mathematics, and Model Physics

CIM

Computer integrated manufacturing

CODEC

Compression/decompression unit, for video compression

$\mathrm{CSCW}$

Computer supported collaborative work

ESNET

DOE's Energy Sciences Network

GCM

Global Climate Modeling

GOSIP

Government Operating System Interconnection Profile

HIPPI

High performance parallel interface

HPCRC

High Performance Computing Research Center

HUGIN

Human Genome Information Network 
ISDN

NREN

OOP

OSI

PCN

RAID

$\mathrm{SCI}$

SCSI

SGML

SMDS

SONET

SQL

$\mathrm{TCP} / \mathrm{IP}$

TDM

Application

Application program

Bit

Broadband

Buffer
Integrated Services Digital Network

National Research and Educational Network

Object oriented programming

Open System Interconnect. A network model by the International Standards Organization

Personal communications network

Redundant array of inexpensive disks

Scalable Coherent Interconnect

Small Computer System Interface

Standard Generalized Markup Language

Switched multi-megabit data service

Synchronous optical network

Structured query language

Transmission Control Protocol/Internal Protocol

Time division multiplexer

\subsection{Definitions}

The specific job to be processed, for example, payroll, accounts receivable, inventory control, or scientific calculation.

Software designed for a specific purpose (such as accounts payable or receivable, payroll, inventory). It may be commercial or DOEdeveloped.

Contraction of binary digit. One of the two states in a binary code.

A communications channel having a bandwidth greater than a voicegrade channel and capable of higher speed data transfer.

A storage area in sending and receiving devices that is used to collect and temporarily store data. The use of buffers compensates for differences in rates of data flow. 
Byte

Circuit switching

Cluster

Compiler

Configuration

Coupler

Database

Database

management system

(DBMS)

Data communications

Data sink

Dedicated channel

Dedicated

communications link
A bit string that is interpreted as a signal unit, usually of eight bits in length.

The temporary direct electrical connection of two or more channels between two or more points to provide a user with exclusive use of an open circuit with which to exchange information.

A group of co-located terminals that share a communications facility via a terminal controller.

Software that translates a high-level language into the computer's machine language, as well as acting as an assembler.

The components which make up an information-processing system.

A device that interconnects a circuit to a terminal. Converts digital signals to analog and vise versa.

A complete, integrated collection of data organized to avoid duplication yet permit retrieval of information to satisfy a wide variety of user information needs. Commonly used to refer to a file or collection of files containing data. Technically, database refers to a technique for designing programming languages such that the logic instructions are separate from the data description. This allows modification of the data description (e.g., adding a field to a file) without modifying all programs using the file.

The software that controls the organizing, cataloging, locating, storing, retrieving, and maintaining of data in a database.

The movement of encoded information (data) through a transmission medium. This includes assembly, sequencing, routing, and selection of such information which is generated at independent remote points of data origination, and the distribution of the processed information to remote output terminals or other data processing equipment.

The equipment that accepts data signals after transmission.

A channel that is leased from a common carrier for the exclusive use of the leaser.

A link that is continuously available to the user. Also called private or leased. 
Distributed network

Distributed

processing

Encryption

Error Detecting Code (EDC)

Flowchart

FORTRAN (FORmula TRANslation)

Gigabyte

High-Level Language

Host Processor

Interactive

LAN (local area network)
A communications network made up of two or more processing centers where processing is evenly distributed between each center and where each processor center is in communication with the other.

A multi-terminal system which shares peripherals and sometimes storage, but where computing power is dispersed among individual stations or systems components.

The process of hiding the information content of a message by the application of a secret algorithm.

A code in which each data signal conforms to specific rules of construction so that departures from this construction in the received signals are automatically detected. Such codes require more signal elements than are necessary to convey the fundamental information.

A documentation method of graphically describing what a program or system is intended to do; used as a blueprint for writing programs and as documentation for programs and systems.

A high-level computer language used primarily for mathematical computations. Although FORTRAN is available for some small computers, it is primarily used with large computer systems. One of the oldest programming languages.

One billion (10 to the ninth) bytes.

A method of programming that allows a person to give instructions to a computer in a procedural, algebraic form or English language form that is problem oriented. A high-level language (e.g., BASIC, FORTRAN) is often considered to be machine independent because it has no direct relationship to a specific machine language.

An information processor that provides supporting services and/or guidance to users and/or to satellite processors, terminals, and other subsidiary devices. A host processor is generally assumed to be selfsufficient and to require no supervision from other processors.

An application in which each entry elicits a response. An interactive system may also be conversational, implying continuous dialog between the user and the system.

A communications network within a city or complex. 
Megabyte

Microprocessor

Multiplexing

Network

Node

Operating system

Packet switching

Packet switching network

Petabyte

Protocol
One million (10 to the sixth) bytes. A sizable novel is about a megabyte of data.

The central processing unit of a microcomputer (usually in a single integrated circuit) which holds all the elements for manipulating data and performing arithmetic calculations.

The division of a transmission facility into two or more channels either by horizontally splitting the frequency band transmitted by the channel into narrower bands, each of which is used to constitute a distinct channel, or by allotting this common channel to several different vertical information channels one at a time.

An interconnected system of computers and/or terminals. The components do not have to be physically close to one another and are often connected by telephone lines.

A station on a network. A node can be a computer or terminal.

A collection of integrated service routines that control the overall operation of a computer system; includes the functions of security, accounting, scheduling, file access, error recovery, and communications.

The transmission of data by means of addressed packets whereby a transmission channel is occupied for the duration of transmission of the packet only. The channel is then available for the transmission of packets between other data terminal equipment.

A network designed to carry data in the form of packets. The packet and its format is internal to the network.

10 to the 15 th bytes (1000 terabytes)-equivalent to about $1,000,000,000$ novels.

The convention by which data are transmitted over a line. Synchronous protocol is a form of transmission that uses no redundant information (such as the start and stop bits in asynchronous transmission) to identify the beginning and end of each character. A bisynchronous protocol is a set of specific operating procedures originated by IBM for the synchronous transmission of binary coded data. Since fewer non-character bits are required for synchronous and bisynchronous transmission, these protocols provide for a more efficient use of line time. 
Real time

Router

Routine

Routing

RS-232C

Software

Switch

Telecommunication

Terabyte

Transparency

VLSI (very-large-scale integration)

X.25
Term used to describe a computer that is processing data fast enough for it to be used to control the operation that is supplying the data.

Boxes which have connections to multiple networks and pass traffic between the nets.

The algorithm by which a network directs a packet from its source to its destination.

The assignment of a communications pathway through a network with multiple possibilities.

A technical specification published by the Electronic Industries Association which specifies one way in which a computer communicates with a peripheral (such as a modem or terminal).

All the programming systems, programs, or segments of programs used to support a computer. The term was coined to contrast with hardware (the actual mechanics and circuitry of a computer).

Telephone company switching facility.

Transmission of data between a computer and another computer or terminal in a different location. It can take place via phone lines, satellites, radio waves, optical fibers, or other means.

10 to the 12 th bytes (1000 gigabytes) -equivalent to about one million novels.

The ability of a communications system to pass control signals or codes as data to the receiving unit without affecting the communications system.

A term used to describe a semiconductor device that has up to 10,000 electronic circuits on a single silicon chip.

A protocol developed by CCITT (the standards writing organization for international telephone carriers) for packet switching procedures. 

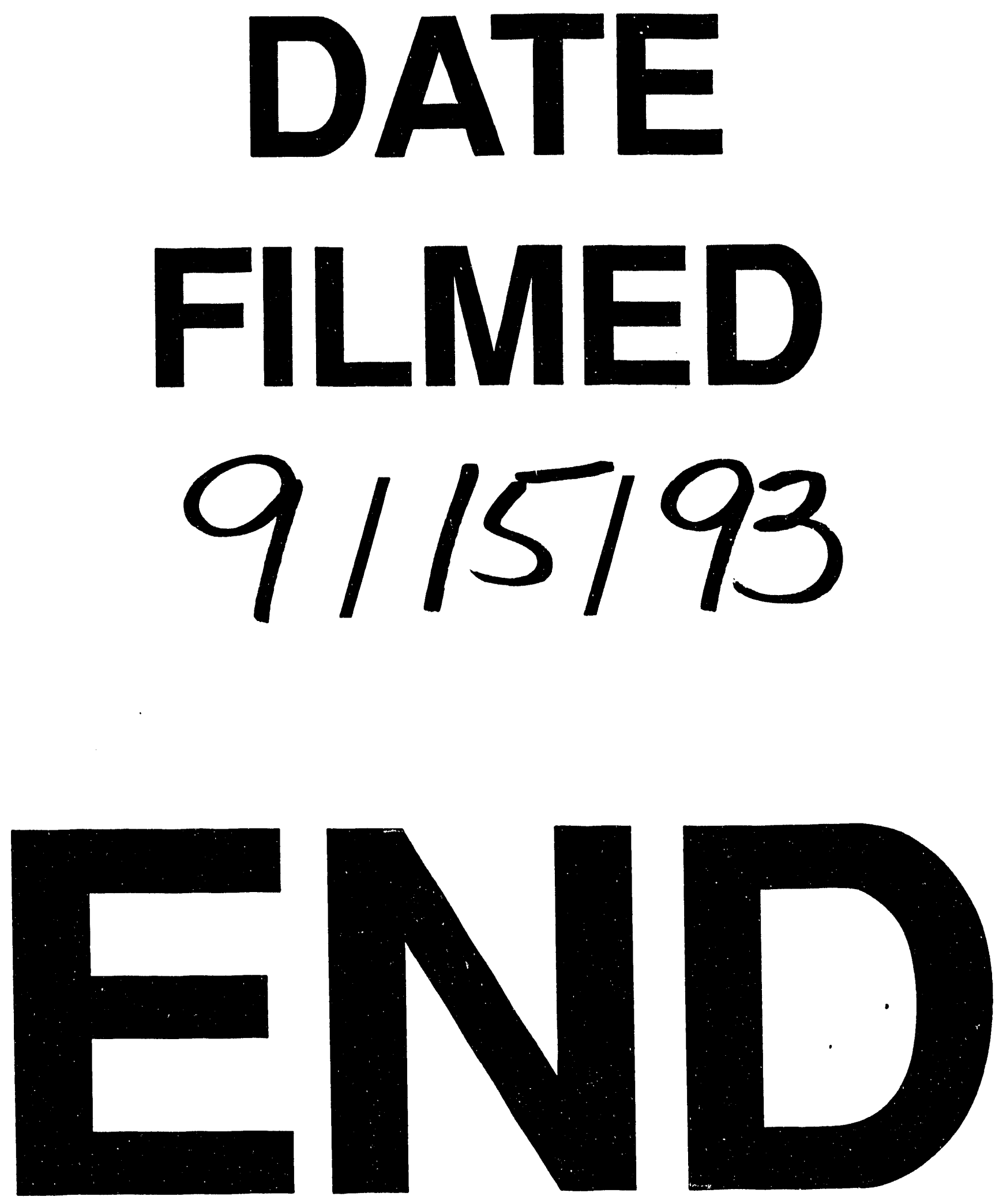
